\title{
Frontal White Matter Tracts Sustaining Speech Production in Primary Progressive Aphasia
}

\author{
Maria Luisa Mandelli, ${ }^{1}$ Eduardo Caverzasi, ${ }^{1,2}$ Richard J. Binney, ${ }^{1}$ Maya L. Henry, ${ }^{1}$ Iryna Lobach, ${ }^{1}$ (D) Nikolas Block, \\ Bagrat Amirbekian, ${ }^{2,3}$ Nina Dronkers, ${ }^{4}$ Bruce L. Miller, ${ }^{1}$ Roland G. Henry, ${ }^{2,3,5}$ and Maria Luisa Gorno-Tempini ${ }^{1}$ \\ ${ }^{1}$ Memory and Aging Center, Department of Neurology, and ${ }^{2}$ Department of Neurology, San Francisco, California 94143 , ${ }^{3}$ Graduate Group in \\ Bioengineering, University of California, Berkeley, California 94720-1762, ${ }^{4}$ Center for Aphasia and Related Disorders, Veterans Administration Northern \\ California Health Care System, Martinez, California 94553, and ${ }^{5}$ Department of Radiology and Biomedical Imaging, University of California, San Francisco, \\ California 94107
}

In primary progressive aphasia (PPA), speech and language difficulties are caused by neurodegeneration of specific brain networks. In the nonfluent/agrammatic variant (nfvPPA), motor speech and grammatical deficits are associated with atrophy in a left fronto-insularstriatal network previously implicated in speech production. In vivo dissection of the crossing white matter (WM) tracts within this "speech production network" is complex and has rarely been performed in health or in PPA. We hypothesized that damage to these tracts would be specific to nfvPPA and would correlate with differential aspects of the patients' fluency abilities. We prospectively studied 25 PPA and 21 healthy individuals who underwent extensive cognitive testing and 3 T MRI. Using residual bootstrap Q-ball probabilistic tractography on high angular resolution diffusion-weighted imaging (HARDI), we reconstructed pathways connecting posterior inferior frontal, inferior premotor, insula, supplementary motor area (SMA) complex, striatum, and standard ventral and dorsal language pathways. We extracted tract-specific diffusion tensor imaging (DTI) metrics to assess changes across PPA variants and perform brainbehavioral correlations. Significant WM changes in the left intrafrontal and frontostriatal pathways were found in nfvPPA, but not in the semantic or logopenic variants. Correlations between tract-specific DTI metrics with cognitive scores confirmed the specific involvement of this anterior-dorsal network in fluency and suggested a preferential role of a posterior premotor-SMA pathway in motor speech. This study shows that left WM pathways connecting the speech production network are selectively damaged in nfvPPA and suggests that different tracts within this system are involved in subcomponents of fluency. These findings emphasize the emerging role of diffusion imaging in the differential diagnosis of neurodegenerative diseases.

Key words: diffusion tensor imaging; frontal tracts; primary progressive aphasia; speech production; tractography; white matter

\section{Introduction}

Primary progressive aphasia (PPA) is a neurodegenerative clinical syndrome characterized by progressive decline in speech and language functions (Mesulam, 1982). Three main clinical variants have been identified on the basis of cognitive features: a

Received Aug. 11, 2013; revised April 29, 2014; accepted June 1, 2014.

Author contributions: M.L.M., E.C., B.A., R.G.H., and M.L.G.-T. designed research; M.L.M., E.C., M.L.H., N.B., B.L.M., and M.L.G.-T. performed research; M.L.M., E.C., R.J.B., B.A., N.D., B.L.M., R.G.H., and M.L.G.-T. contributed unpublished reagents/analytic tools; M.L.M., M.L.H., and I.L. analyzed data; M.L.M., E.C., R.J.B., M.L.H., and M.L.G.-T. wrote the paper.

The study was supported by grants from the National Institutes of Health (NINDS R01 NS050915, NIA P50 AG03006, NIA P50 AG023501, NIA P01 AG019724), State of California (DHS04-35516), Alzheimer's Disease Research Centre of California (03-75271 DHS/ADP/ARCC), Larry L. Hillblom Foundation, John Douglas French Alzheimer's Foundation, Koret Family Foundation, Consortium for Frontotemporal Dementia Research, and McBean Family Foundation. These supporting sources had no involvement in the study design, collection, analysis or interpretation of data, nor were they involved in writing the paper or the decision to submit this report for publication. We thank the patients and their families for the time and effort they dedicated to the research. We thank Minerva Meese for her help in speech evaluation.

The authors declare no competing financial interests.

Correspondence should be addressed to Maria Luisa Mandelli, UCSF Memory and Aging Center, Department of Neurology, University of California San Francisco, Sandler Neurosciences Center, 675 Nelson Rising Lane, Suite 190, Box 1207, San Francisco, CA 94143-1207. E-mail: MariaLuisa.Mandelli@ucsf.edu.

DOI:10.1523/JNEUROSCI.3464-13.2014

Copyright $\odot 2014$ the authors $\quad 0270-6474 / 14 / 349754-14 \$ 15.00 / 0$ nonfluent/agrammatic variant (nfvPPA), a semantic variant (svPPA), and a logopenic variant (lvPPA) (Gorno-Tempini et al., 2004b). Motor speech and grammatical deficits resulting in speech production difficulties are typical features of nfvPPA; however, diagnosis purely on the basis of linguistic symptoms can be difficult, especially between the nonfluent/agrammatic and logopenic variants, which are both characterized by speech output errors (Ogar et al., 2007; Gorno-Tempini et al., 2008; Grossman, 2012; Josephs et al., 2012).

Neuroimaging is helpful in the differentiation of the PPA variants. Accordingly, the most recent international classification scheme includes an "imaging-supported" diagnosis, indicating specific regional gray matter (GM) changes for each subtype. In nfvPPA, GM damage is typically found in left inferior frontal, insular, supplementary motor, and striatal regions (Grossman et al., 1996; Nestor et al., 2003; Gorno-Tempini et al., 2006), suggesting that this "speech production network" is responsible for the fluency deficits typical of this variant (Wilson et al., 2010), as was observed previously in aphasia due to stroke (Broca, 1865; Dronkers, 1996). Recent studies have demonstrated the presence of white matter (WM) damage in the PPA variants using diffusion tensor imaging (DTI). Most investigations focused on the 
standard fronto-temporo-parietal dorsal and ventral language pathways (Borroni et al., 2007; Whitwell et al., 2010; Galantucci et al., 2011; Agosta et al., 2012a; Grossman et al., 2013; Mahoney et al., 2013; Sajjadi et al., 2013; Schwindt et al., 2013) and have found damage to the superior longitudinal fasciculus in both nfvPPA and lvPPA, although changes were more widespread in nfvPPA (Galantucci et al., 2011). Because GM changes in nfvPPA occur mainly within the left fronto-insular-striatal speech production network, we hypothesized that damage to the WM tracts connecting these areas would be specifically damaged in this variant. These intrafrontal and frontostriatal tracts have not been studied extensively, likely because of methodological difficulties related to the high density of crossing fibers in these regions. One previous investigation showed damage of an intrafrontal bundle, called the frontal aslant tract, in nfvPPA (Catani et al., 2013). To our knowledge, no previous tractography study has investigated the WM pathways connecting the entire speech production network in PPA.

Here, we applied residual bootstrap Q-ball tractography for in vivo dissection of WM tracts connecting the fronto-insular cortical and subcortical speech production network in PPA bilaterally. This methodology has the capability of quantifying multiple intravoxel fiber orientations, thus resolving crossing fibers and reducing false negatives in the reconstruction of WM tracts (Berman et al., 2008). Tract-specific DTI metrics were compared across PPA variants and correlated with speech and language scores. We hypothesized that significant WM injury in the speech production network would occur in nfvPPA only and that the severity of damage in specific tracts would correlate with different aspects of fluency.

\section{Materials and Methods}

\section{Participants and diagnostic assessment}

A total of 25 patients with sporadic PPA ( 9 nfvPPA, 3 male $/ 6$ female; 8 svPPA, 4 male/ 4 female; and 8 lvPPA, 4 male/ 4 female) and 21 healthy individuals ( 8 male/13 female) matched for age and sex were evaluated at the Memory and Aging Center (MAC) at the University of CaliforniaSan Francisco (UCSF). All subjects were right-handed and gave written informed consent. The study was approved by the UCSF institutional review board. PPA patients were divided into clinical variants applying current international guidelines for diagnosis (Gorno-Tempini et al., 2011). We considered clinical data obtained at first evaluation at the UCSF MAC and 3 T MRI acquired within 6 months of such a visit. Clinical evaluation included a semistructured history and physical examination, caregiver interview, standardized neuropsychological screening, and a detailed speech and language evaluation, as described previously (Gorno-Tempini et al., 2004b). All patients reported a negative family history of PPA.

We performed a Pearson $\chi^{2}$ test or univariate ANOVA for demographics and speech-language test scores to show expected differences among PPA variants (Table 1).

\section{MRI}

Subjects were scanned on a Siemens TrioTim syngo 3 T MR scanner equipped with an eight-channel head coil. The MRI protocol included a high-resolution 3D T1-weighted image with the following parameters: 160 sagittal slices, with $1 \mathrm{~mm}$ thickness; repetition time (TR)/echo time $(\mathrm{TE})=2300 / 2.98 \mathrm{~ms}$; inversion time $=900 \mathrm{~ms}$; flip angle $=9^{\circ}$; field of view $=256 \mathrm{~mm}^{2}$; matrix $=256 \times 240$; voxel size $1 \mathrm{~mm}^{3}$ isotropic. A high angular resolution diffusion-weighted imaging (HARDI) dataset was acquired using a single-shot spin-echo echo-planar imaging (SE-EPI) sequence including 55 contiguous axial slices acquired in an interleaved order with in-plane resolution $=2.2 \mathrm{~mm}^{2}$, slice thickness $=2.2 \mathrm{~mm}$, $\mathrm{TR} / \mathrm{TE}=8000 / 109 \mathrm{~ms}$, flip angle $=90^{\circ}$, matrix $=100 \times 100,64$ noncollinear diffusion sensitization directions at $b=2000 \mathrm{~s} / \mathrm{mm}^{2}, 1$ at $b=0$, and an integrated parallel acquisition technique acceleration factor of 2 .
Table 1. Demographic and clinical data of the subjects

\begin{tabular}{lllll}
\hline & HC & NfvPPA & SvPPA & LvPPA \\
\hline Age & $65.3(3.9)$ & $67.3(5.7)$ & $61.4(5.6)$ & $63.6(7.1)$ \\
Sex (F/M) & $13 / 8$ & $6 / 3$ & $5 / 3$ & $4 / 4$ \\
Education & $17.3(2.2)$ & $15.1(2.8)$ & $16.0(1.5)$ & $16.1(3.2)$ \\
Disease duration & NA & $4.2(1.8)$ & $5.4(3.2)$ & $5.1(4.4)$ \\
MMSE (30) & $29.5(0.7)$ & $25.1(3.8)^{*}$ & $24.4(5.1)^{*}$ & $23.8(6.4)^{*}$ \\
CDR & NA & $0.5(0.4)$ & $0.8(0.5)$ & $0.6(0.2)$ \\
Speech and language evaluation & & & & \\
$\quad$ WAB-Spontaneous Speech & $0.0(0.0)$ & $6.1(1.8)^{*, b}$ & $8.6(0.9)$ & $8.1(1.5)$ \\
$\quad$ Fluency (10) & & & & \\
$\quad$ WAB Rep Tot (100) & NA & $78.5(10.2)$ & $84.1(17.9)$ & $71.8(17.1)$ \\
$\quad$ Boston Naming Test (15) & $14.6(0.5)$ & $12.2(2.0)$ & $6.2(5.8)^{* a, c}$ & $9.6(5.0)^{*}$ \\
$\quad$ MSE Apraxia of Speech & $0.0(0.0)$ & $2.9(1.3)^{* b, c}$ & $0.0(0.0)$ & $0.5(1.1)$ \\
$\quad$ Rating (0-7) & & & & \\
$\quad$ MSE Dysarthria Rating (0-7) & $0.0(0.0)$ & $2.1(2.1)^{* b}$ & $0.0(0.0)$ & $0.38(1.1)$ \\
$\quad \begin{array}{l}\text { Speech Production Score (1) } \\
\text { WABSequential Commands(80) }\end{array}$ & $0.0(0.0)$ & $0.63(0.1)^{b, c}$ & $0.92(0.1)$ & $0.87(0.1)$ \\
Pyramids and Palm & NA & $41.3(8.5)$ & $65.3(18.3)$ & $68.3(11.8)$ \\
$\quad$ Trees-Picture Version (52) & & & & \\
$\quad$ Peabody Picture Vocabulary & NA & $14.3(1.3)$ & $41.3(7.2)^{a, c}$ & $50.4(1.2)$ \\
$\quad$ Test-Revised (16) & & & & \\
Other cognitive domains & & & & \\
$\quad$ Benson Figure Copy (17) & $15.0(1.4)$ & $14.9(1.8)$ & $15.6(0.9)$ & $14.9(1.1)$ \\
$\quad$ Benson Figure Delay Recall (17) & $11.5(2.8)$ & $9.3(3.5)$ & $7.0(5.2)^{*}$ & $7.3(2.9)$ \\
$\quad$ Digit Span Backwards & $6.1(1.4)$ & $3.1(1.3)^{* b}$ & $5.1(1.1)$ & $3.3(1.0)^{* b}$ \\
\hline
\end{tabular}

Values are reported as mean (SD). * Significant differences compared with controls at $p<0.05$.

${ }^{a}$ Significant differences from nonfluent variants at $p<0.05$.

${ }^{b}$ Significant differences from semantic variants at $p<0.05$.

'Significant differences from logopenic variants at $p<0.05$.

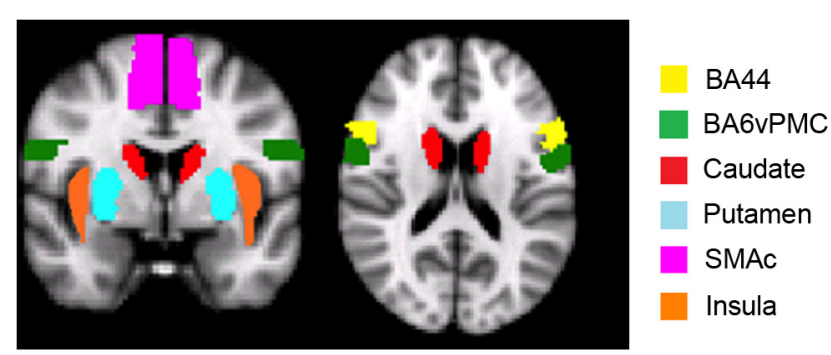

Figure 1. ROls used for tractography to define the speech production network are displayed in MNI space.

\section{Diffusion imaging data preprocessing}

Initial image preprocessing was performed using the FMRIB Software Library (FSL; http://www.fmrib.ox.ac.uk/fsl/). For each subject, T1weighted and diffusion-weighted images (DWIs) were skull stripped using FSL's brain extraction tool. The T1 image was registered (rigid body alignment) to the $b_{0}$ volume of the DWI dataset and subsequently registered to the Montreal Neurological Institute (MNI) standard stereotactic atlas using FSL's linear and nonlinear registration tool. Preprocessing of the DWI datasets was performed using FSL's diffusion toolbox. Eddy current distortions and motion artifacts were corrected by registering each diffusion-sensitized volume to the $b_{0}$ volume with an affine transform. After tensor diagonalization, whole-brain maps of voxelwise quantitative WM metrics such as mean diffusivity (MD), fractional anisotropy (FA), axial diffusivity (AD) and radial diffusivity (RD) were obtained. These maps were warped into MNI space using the transform estimated for the coregistered T1 image.

\section{Regions of interest for the speech production network}

The main aim of our study was to investigate the WM pathways connecting cortical and subcortical GM regions within the speech production network known to be atrophied in nfvPPA, such as Brodmann area 44 (BA44), ventral premotor cortex (BA6vPMC), the supplementary motor 
area complex (SMAc), the insula, and the striatum (Gorno-Tempini et al., 2004a; GornoTempini et al., 2006; Rogalski et al., 2011a; Grossman, 2012; Grossman et al., 2013). These regions have also been shown to be involved in speech production in fMRI studies of healthy subjects (Eickhoff et al., 2009), in neuropsychological studies of stroke patients, and in intraoperative mapping (Broca, 1865; Dronkers, 1996; Henry et al., 2004; Hillis et al., 2004; Wilson et al., 2009). We defined specific regions of interest (ROIs) as seeds and targets for tractography in the following manner. For ventral premotor BA6 we considered only the face/mouth area (BA6vPMC, 5 voxels centered at $-58 / 1 / 23$ in MNI space) as defined by both fMRI and intraoperative mapping studies (Henry et al., 2004; Eickhoff et al., 2009). For BA44, we used the Juelich Histological Atlas. For the SMAc, the insular cortex, and the striatum (caudate and putamen), we used the Harvard-Oxford Atlas (http://fsl.fmrib.ox.ac.uk/fsl/fsl4.0/fslview/ atlas-descriptions.html). All ROIs were defined in both hemispheres (Fig. 1). To ensure that patients with nfvPPA included in this study showed GM atrophy consistent with pre-

vious studies, we conducted a voxel-based morphometry (VBM) analysis. The analysis was performed using the SPM8 software package (http://www.fil.ion.ucl.ac.uk/spm) and a diffeomorphic exponentiated lie algebra (DARTEL) registration method (Ashburner, 2007).

We also tracked the most commonly described ventral and dorsal language-related pathways previously involved in PPA (Borroni et al., 2007; Whitwell et al., 2010; Galantucci et al., 2011; Agosta et al., 2012b; Grossman, 2012; Mahoney et al., 2013; Schwindt et al., 2013): the superior longitudinal fasciculus (SLF II/III), the arcuate fasciculus (AF), the inferior frontal-occipital fasciculus (IFOF), the uncinate fasciculus (UF), the inferior longitudinal fasciculus (ILF), and its anterior part in particular (at $y=-8$ on coronal plane). The ROIs used to delineate these tracts were described previously (Galantucci et al., 2011). All ROIs were defined in MNI space and subsequently warped to each healthy control subject's native DWI space for tractography. A schema of the expected connections in the left hemisphere is shown in Figure 2.

\section{Residual bootstrap Q-ball tractography}

We reconstructed all tracts with a probabilistic streamline tractography algorithm in combination with a residual bootstrap Q-ball imaging method (Berman et al., 2008) developed in Dipy (http://nipy.sourceforge. net/dipy/). Starting ROIs were densely seeded with $7^{3}$ points equally spaced throughout each voxel. Streamline propagation continued until reaching a voxel with $\mathrm{FA}<0.15$ or a turning angle $>60^{\circ}$. Tracts for the fronto-insular-striatal connections were reconstructed in both hemispheres using a two-ROI seed-target approach. The general procedure involved reconstructing tracts in healthy subjects in order to create mean templates that could be used to extract tract-specific DTI metrics in each patient. This was necessary because templates for the fronto-insularstriatal tracts are not included in standard software packages or anatomical atlases.

We then converted these tracts into density maps (indicating how many streamlines pass through a voxel) and normalized them to MNI space. Templates for each tract were then created by calculating the mean of the density maps. Finally, binary maps were produced and warped back into each subject's native DWI space for both healthy controls and PPA cohorts. From these masks, we extracted the median of the DTI metrics of each tract in each subject.

\section{Statistical analysis: group comparison of DTI metrics}

We performed a univariate ANCOVA model to test differences in each DTI metric between PPA variants and healthy controls for each WM tract. To correct for multiple comparisons among groups, we performed

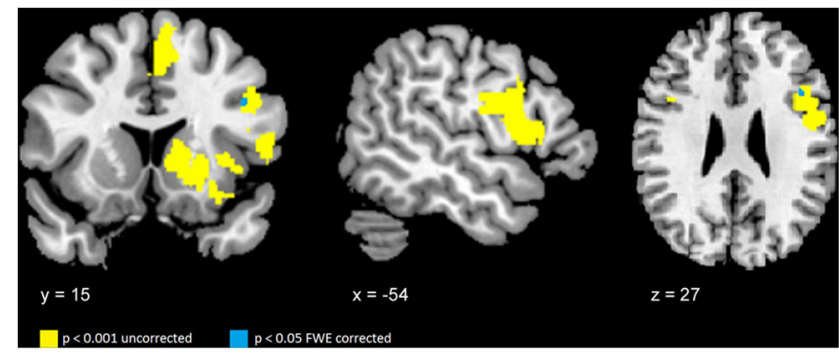

Figure 3. VBM in nfvPPA compared with controls. The figure illustrates the regions correspond to the pre-SMA and SMAC, BA44, the ventral premotor cortex (BA6), the left insula, and the left striatum (yellow). Only a cluster in left the inferior frontal gyrus (blue) survives after familywise error (FWE) correction at $p<0.05$.

a post hoc Tukey honest significant error test. Age, sex, and total intracranial volume (as calculated using SPM8 within the VBM procedure) were included as nuisance covariates. Because we considered 27 tracts for each subject, we used a further Bonferroni correction for 27 comparisons relative to controls. The resulting threshold of statistical significance was thus $p<(0.05 / 27)=0.002$.

We also compared nfvPPA with the other two PPA variants. We performed this comparison only in tracts where significant differences were found in nfvPPA versus healthy controls. Because of the small number of subjects included in this analysis and the known greater variability within patient groups, we did not correct this analysis for multiple comparisons.

\section{Statistical analysis: correlation analyses between cognitive scores and tract-specific FA values}

To assess brain behavioral associations, we performed Spearman correlations between FA values (as an index of WM integrity) and specific cognitive scores. Statistical analyses were performed using R (http://www. r-project.org/). We only considered tracts that showed significant changes in nfvPPA versus controls in all DTI metrics at our threshold ( $p$ $<0.002$ ).

We performed two analyses to evaluate the specificity of the speech production tracts in fluency in the whole PPA group and the differential contributions of frontal speech and standard language tracts to different components of the fluency construct in the nfvPPA group alone (i.e., motor speech, speech rate, sentence comprehension, and production). 


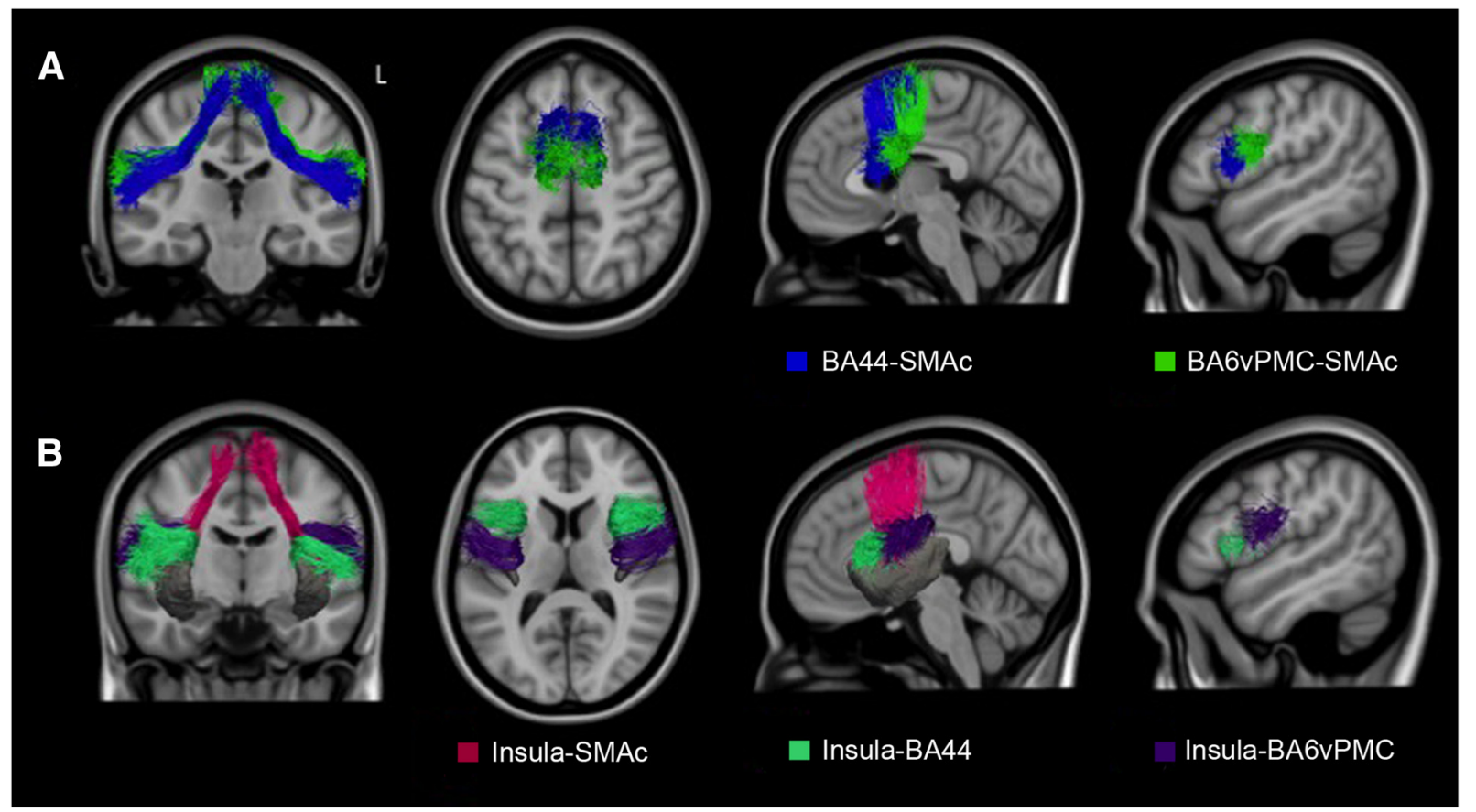

Figure 4. 3D representation of the fronto-cortical-insular speech production tracts. $A$, Tracts connecting the pre-SMA and SMA to BA44 (blue) and the ventral premotor cortex (BA6; green). $\boldsymbol{B}$, Tracts connecting the insula to the pre-SMA and SMA (pink), insula to BA44 (green), and insula to the ventral premotor cortex (violet).

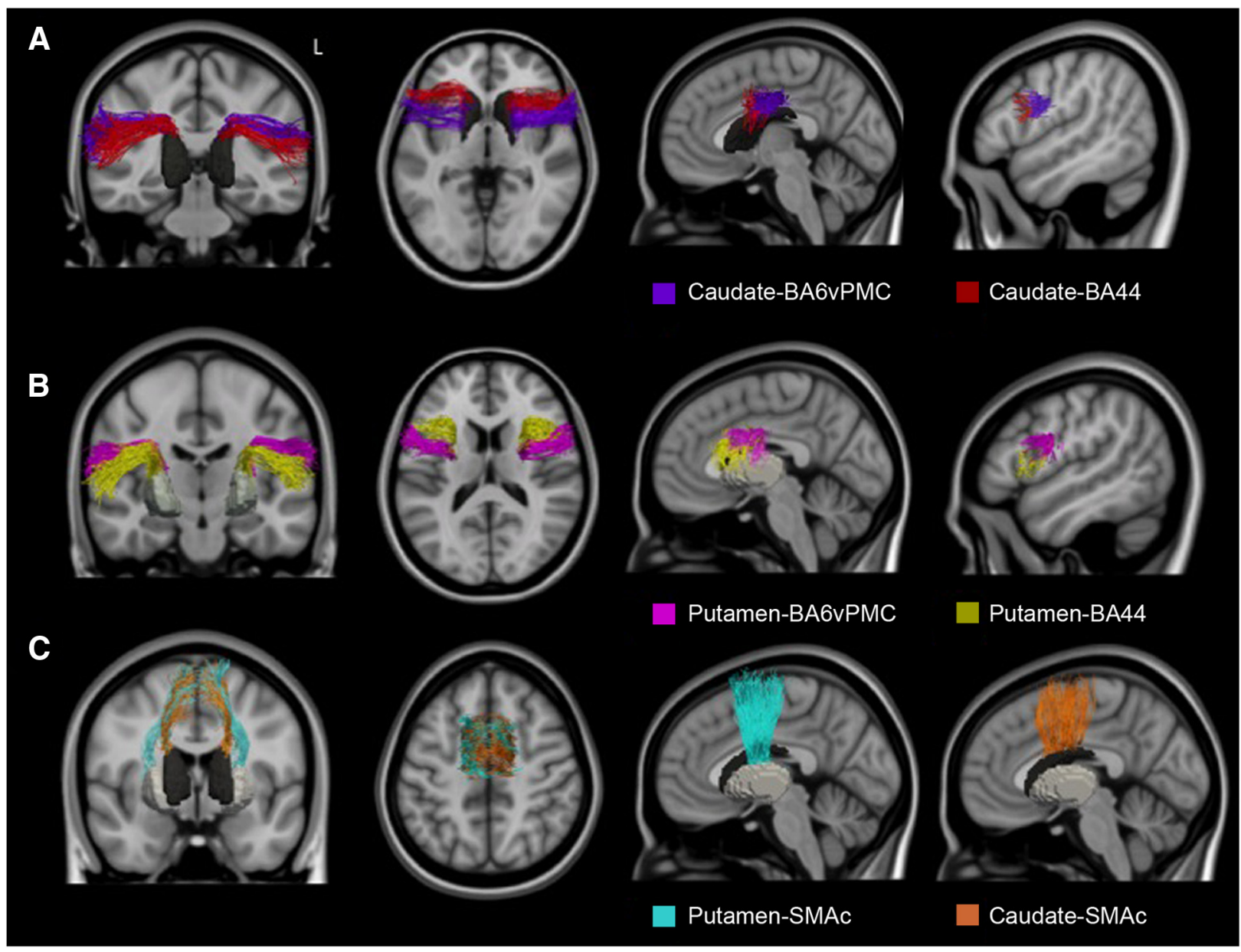

Figure 5. 3D representation of the corticostriatal speech production tracts. $A$, Tracts connecting the caudate to the ventral premotor cortex (BA6; pink) and BA44 (yellow). $B$, Tracts connecting the putamen to the ventral premotor cortex (BA6) (purple) and BA44 (red), the pre-SMA and SMA to the caudate (orange), and the putamen (teal). 


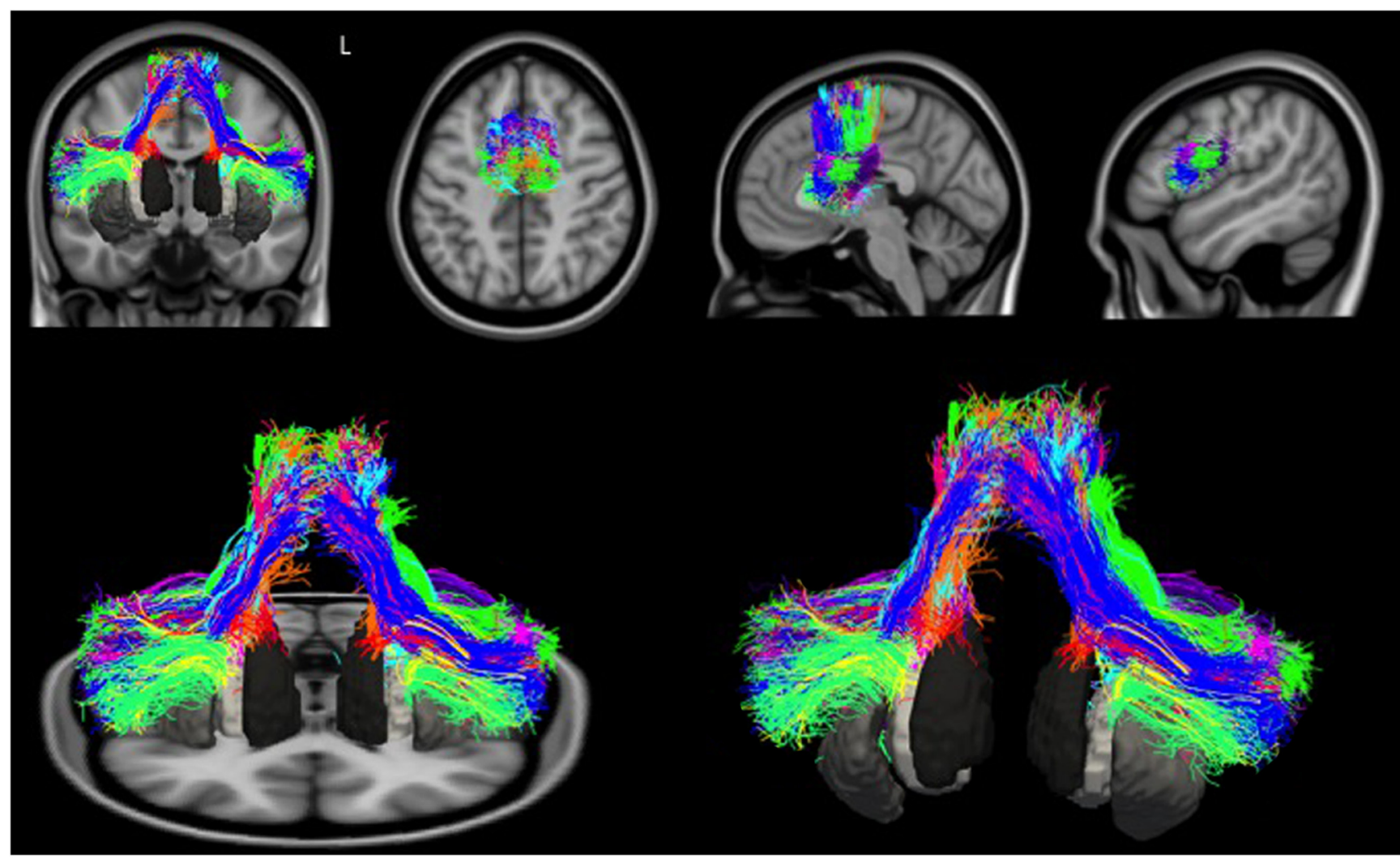

Figure 6. 3D representation of the whole frontal speech production network. All of the tracts reconstructed in the healthy controls are displayed in MNI space with the same colors described for Figure 5.

Specificity of the frontal speech versus ventral tracts in production and comprehension in PPA. For this analysis, we calculated a composite "speech production score" (SP) that could provide a measure of general speech production abilities in all PPA patients. The score was obtained by weighted averaging of the Western Aphasia Battery's Spontaneous Speech (SS-WAB; Kertesz, 1980) score and the Motor Speech Evaluation (MSE; Wertz et al., 1984) score. These tests capture complementary aspects of speech production in structured (MSE) or naturalistic (SS-WAB) conditions. In the MSE, two ratings for apraxia of speech $(\mathrm{AOS})$ and dysarthria are derived on a 7 point scale $(0=$ normal; $7=$ severely impaired), with the most impaired total score being 14 . We did not use the MSE scores alone because svPPA and most lvPPA cases have a score of 0 . The SS-WAB is a comprehensive fluency rating derived from a picture description task on a 10 -point scale $(0=$ no words produced; $10=$ sentences of normal length without articulatory difficulty or speech sound errors). The SP score was calculated applying the following formula: $\mathrm{SP}=\mathrm{SS} / 10+((14-\mathrm{MSE}) / 14) / 2$, in which $\mathrm{SP}=0$ indicates maximal impairment. For language comprehension and semantics, we considered scores from a visual semantic association task (Pyramids and Palm Trees Test-Picture Version) and a words-to-picture matching task (Peabody Picture Vocabulary Test Revised). We correlated SP and comprehension scores with FA values in frontal speech tracts and ILF pathway. We hypothesized that the frontal speech tracts and ILF would show selective correlations with SP or comprehension scores, respectively.

Differential contribution of speech versus dorsal language tracts to the cognitive components of fluency. We considered as components of fluency motor speech (number of distortions), speech rate (number of words per minute), syntax production (number of words in sentences), and sentence comprehension (WAB sequential commands). Distortions of speech sounds are useful in distinguishing individuals with AOS from those with production difficulties arising from phonological impairment. As a proxy for AOS, we counted the number of distorted speech sounds produced per hundred words during the WAB spoken picture description task (number of distortions per hundred words), as described previously (Wilson et al., 2010). Distortions were defined as motor speech errors that involved phonetic alterations in production (e.g., slurring) of a sound. For individuals with dysarthria, only distortions above and beyond the baseline level of production difficulty were counted as distortions. As a proxy for syntax production abilities, we divided the number of words produced in utterances that were sentences by the total number of words produced in the WAB picture description. This variable captures the agrammatic pattern of producing primarily noun phrases and verb phrases in isolation without proper syntactic context (Wilson et al., 2010). Words per minute were also derived from the WAB spoken picture description. The score on the WAB sequential command task was used as a measure of sentence comprehension abilities. We correlated these cognitive measures with FA in frontal speech and in dorsal language tracts (SLF and AF) that have been demonstrated previously to be important for fluency and grammar (Wilson et al., 2011).

\section{Results}

\section{Demographic and cognitive data}

Demographic, clinical, and language data are reported in Table 1. Cognitive and language profiles showed the group differences expected on the basis of clinical diagnosis (Gorno-Tempini et al., 2004b). SS-WAB, MSE, and our composite SP score were significantly impaired in nfvPPA patients compared with the other variants.

Figure 3 illustrates the results of the VBM analysis demonstrating that our nfvPPA cohort showed GM atrophy consistent with previous studies and including our ROIs (Gorno-Tempini et al., 2004a; Grossman, 2011; Rogalski et al., 2011b). All regions showed GM atrophy versus controls at an uncorrected level of significance $p<0.001$, whereas only in a small cluster within the 
Table 2. Group differences in the frontal speech production tracts for all DTI metrics

\begin{tabular}{|c|c|c|c|c|c|c|c|}
\hline \multirow[b]{2}{*}{ Frontal tracts } & \multicolumn{7}{|l|}{ DTI metrics } \\
\hline & $\mathrm{HC}$ & NfvPPA & $p$ & SVPPA & $p$ & LvPPA & $p$ \\
\hline \multicolumn{8}{|l|}{ BA44-SMAC } \\
\hline \multicolumn{8}{|l|}{ Left } \\
\hline$M D$ & $0.600(0.021)$ & $0.677(0.037)^{*, b, c}$ & 0.000 & $0.631(0.039)$ & 0.08 & $0.630(0.024)$ & 0.07 \\
\hline$A D$ & $0.861(0.024)$ & $0.924(0.040)^{*}$ & 0.000 & $0.900(0.045)$ & 0.08 & $0.893(0.022)$ & 0.07 \\
\hline $\mathrm{RD}$ & $0.472(0.024)$ & $0.552(0.038)^{*, b, c}$ & 0.000 & $0.479(0.043)$ & 0.20 & $0.496(0.030)$ & 0.24 \\
\hline MD & $0.587(0.020)$ & $0.637(0.042)^{*}$ & 0.000 & $0.617(0.026)$ & 0.07 & $0.598(0.030)$ & 0.79 \\
\hline$A D$ & $0.844(0.021)$ & $0.877(0.045)$ & 0.38 & $0.878(0.025)$ & 0.06 & $0.860(0.036)$ & 0.56 \\
\hline $\mathrm{RD}$ & $0.458(0.024)$ & $0.516(0.043)^{*, c}$ & 0.000 & $0.487(0.034)$ & 0.12 & $0.466(0.032)$ & 0.93 \\
\hline \multicolumn{8}{|c|}{ BA6vPMC-SMAC } \\
\hline \multicolumn{8}{|c|}{ Left } \\
\hline FA & $0.384(0.022)$ & $0.331(0.035)^{*, b, c}$ & 0.000 & $0.382(0.032)$ & 0.99 & $0.378(0.029)$ & 0.95 \\
\hline MD & $0.579(0.022)$ & $0.639(0.040)^{*}$ & 0.000 & $0.609(0.030)$ & 0.06 & $0.590(0.027)$ & 0.77 \\
\hline$A D$ & $0.839(0.025)$ & $0.900(0.049)^{*}$ & 0.000 & $0.884(0.029)$ & 0.01 & $0.856(0.032)$ & 0.59 \\
\hline $\mathrm{RD}$ & $0.446(0.026)$ & $0.509(0.041)^{*}$ & 0.000 & $0.473(0.033)$ & 0.16 & $0.453(0.028)$ & 0.95 \\
\hline \multicolumn{8}{|l|}{ BA44-Caudate } \\
\hline \multicolumn{8}{|l|}{ Left } \\
\hline FA & $0.324(0.019)$ & $0.292(0.030)$ & 0.01 & $0.305(0.027)$ & 0.25 & $0.303(0.028)$ & 0.20 \\
\hline MD & $0.628(0.018)$ & $0.710(0.036)^{*}$ & 0.000 & $0.672(0.057)$ & 0.01 & $0.653(0.035)$ & 0.26 \\
\hline$A D$ & $0.855(0.021)$ & $0.937(0.040)^{*}$ & 0.000 & $0.901(0.059)$ & 0.02 & $0.880(0.033)$ & 0.34 \\
\hline $\mathrm{RD}$ & $0.512(0.021)$ & $0.591(0.041)^{*}$ & 0.000 & $0.559(0.059)$ & 0.01 & $0.540(0.035)$ & 0.24 \\
\hline \multicolumn{8}{|l|}{ Right } \\
\hline FA & $0.335(0.023)$ & $0.310(0.023)$ & 0.04 & $0.319(0.027)$ & 0.37 & $0.342(0.017)$ & 0.87 \\
\hline MD & $0.608(0.020)$ & $0.657(0.037)^{*}$ & 0.000 & $0.631(0.029)$ & 0.16 & $0.620(0.024)$ & 0.67 \\
\hline$A D$ & $0.834(0.019)$ & $0.888(0.047)^{*}$ & 0.000 & $0.862(0.040)$ & 0.13 & $0.855(0.028)$ & 0.34 \\
\hline FA & $0.291(0.018)$ & $0.273(0.016)$ & 0.33 & $0.286(0.029)$ & 0.44 & $0.292(0.022)$ & 0.90 \\
\hline MD & $0.623(0.021)$ & $0.652(0.031)$ & 0.02 & $0.637(0.026)$ & 0.48 & $0.636(0.023)$ & 0.58 \\
\hline$A D$ & $0.834(0.024)$ & $0.857(0.032)$ & 0.14 & $0.835(0.025)$ & 0.99 & $0.850(0.026)$ & 0.45 \\
\hline RD & $0.517(0.020)$ & $0.549(0.030)$ & 0.01 & $0.536(0.031)$ & 0.25 & $0.522(0.027)$ & 0.95 \\
\hline BA6vPMC-Cau & & & & & & & \\
\hline Left & & & & & & & \\
\hline FA & $0.363(0.027)$ & $0.333(0.016)$ & 0.01 & $0.369(0.017)$ & 0.90 & $0.375(0.023)$ & 0.61 \\
\hline$M D$ & $0.591(0.019)$ & $0.674(0.043)^{*}$ & 0.000 & $0.607(0.033)$ & 0.58 & $0.612(0.028)$ & 0.31 \\
\hline$A D$ & $0.828(0.021)$ & $0.928(0.061)^{*}$ & 0.000 & $0.862(0.058)$ & 0.17 & $0.878(0.032)$ & 0.02 \\
\hline $\mathrm{RD}$ & $0.473(0.023)$ & $0.543(0.037)^{*}$ & 0.000 & $0.478(0.024)$ & 0.96 & $0.488(0.026)$ & 0.51 \\
\hline Right & & & & & & & \\
\hline FA & $0.344(0.019)$ & $0.332(0.018)$ & 0.45 & $0.342(0.027)$ & 0.99 & $0.349(0.022)$ & 0.96 \\
\hline MD & $0.599(0.018)$ & $0.654(0.041)^{*}$ & 0.000 & $0.620(0.029)$ & 0.22 & $0.608(0.026)$ & 0.83 \\
\hline$A D$ & $0.822(0.017)$ & $0.899(0.058)^{*}$ & 0.000 & $0.856(0.049)$ & 0.11 & $0.846(0.029)$ & 0.40 \\
\hline $\mathrm{RD}$ & $0.481(0.021)$ & $0.533(0.036)^{*}$ & 0.000 & $0.499(0.030)$ & 0.37 & $0.488(0.026)$ & 0.92 \\
\hline BA6vPMC-Puta & & & & & & & \\
\hline Left & & & & & & & \\
\hline FA & $0.299(0.027)$ & $0.265(0.025)$ & 0.005 & $0.300(0.020)$ & 1.00 & $0.297(0.019)$ & 0.99 \\
\hline$M D$ & $0.629(0.022)$ & $0.687(0.036)^{*}$ & 0.000 & $0.632(0.033)$ & 0.99 & $0.648(0.036)$ & 0.44 \\
\hline$A D$ & $0.849(0.029)$ & $0.897(0.029)$ & 0.002 & $0.851(0.029)$ & 0.99 & $0.871(0.040)$ & 0.33 \\
\hline $\mathrm{RD}$ & $0.519(0.024)$ & $0.581(0.041)^{*}$ & 0.000 & $0.525(0.032)$ & 0.95 & $0.536(0.03)$ & 0.54 \\
\hline Right & & & & & & & \\
\hline FA & $0.321(0.016)$ & $0.303(0.026)$ & 0.20 & $0.315(0.027)$ & 0.92 & $0.326(0.028)$ & 0.94 \\
\hline$M D$ & $0.619(0.023)$ & $0.650(0.030)$ & 0.03 & $0.628(0.025)$ & 0.86 & $0.623(0.035)$ & 0.99 \\
\hline$A D$ & $0.844(0.029)$ & $0.870(0.030)$ & 0.13 & $0.850(0.031)$ & 0.95 & $0.857(0.029)$ & 0.68 \\
\hline $\mathrm{RD}$ & $0.500(0.017)$ & $0.533(0.030)$ & 0.01 & $0.516(0.028)$ & 0.42 & $0.507(0.034)$ & 0.92 \\
\hline
\end{tabular}


Table 2. Continued

\begin{tabular}{|c|c|c|c|c|c|c|c|}
\hline \multirow[b]{2}{*}{ Frontal tracts } & \multicolumn{7}{|l|}{ DTI metrics } \\
\hline & $\mathrm{HC}$ & NfyPPA & $p$ & SvPPA & $p$ & LVPPA & $p$ \\
\hline \multicolumn{8}{|l|}{ SMAc-Caudate } \\
\hline \multicolumn{8}{|l|}{ Left } \\
\hline FA & $0.364(0.028)$ & $0.306(0.032)^{*, b}$ & 0.000 & $0.346(0.037)$ & 0.48 & $0.338(0.027)$ & 0.17 \\
\hline MD & $0.610(0.020)$ & $0.698(0.050)^{*, b, c}$ & 0.000 & $0.646(0.046)$ & 0.06 & $0.641(0.027)$ & 0.10 \\
\hline$A D$ & $0.865(0.021)$ & $0.940(0.056)^{*, c}$ & 0.000 & $0.904(0.045)$ & 0.06 & $0.893(0.023)$ & 0.23 \\
\hline $\mathrm{RD}$ & $0.481(0.027)$ & $0.576(0.050)^{*, b, c}$ & 0.000 & $0.517(0.051)$ & 0.09 & $0.515(0.032)$ & 0.12 \\
\hline \multicolumn{8}{|l|}{ Right } \\
\hline FA & $0.352(0.025)$ & $0.304(0.020)^{*, c}$ & 0.000 & $0.327(0.029)$ & 0.07 & $0.345(0.023)$ & 0.90 \\
\hline MD & $0.613(0.020)$ & $0.678(0.043)^{*, c}$ & 0.000 & $0.641(0.029)$ & 0.09 & $0.625(0.025)$ & 0.71 \\
\hline$A D$ & $0.862(0.019)$ & $0.916(0.048)^{*}$ & 0.000 & $0.889(0.026)$ & 0.12 & $0.880(0.032)$ & 0.45 \\
\hline $\mathrm{RD}$ & $0.486(0.026)$ & $0.559(0.042)^{*, b, c}$ & 0.000 & $0.518(0.035)$ & 0.08 & $0.500(0.028)$ & 0.71 \\
\hline \multicolumn{8}{|l|}{ SMAc-Putamen } \\
\hline \multicolumn{8}{|l|}{ Left } \\
\hline FA & $0.337(0.025)$ & $0.308(0.036)$ & 0.01 & $0.350(0.038)$ & 0.74 & $0.334(0.033)$ & 0.99 \\
\hline MD & $0.608(0.021)$ & $0.678(0.040)^{*, b, c}$ & 0.000 & $0.631(0.041)$ & 0.27 & $0.637(0.030)$ & 0.10 \\
\hline$A D$ & $0.847(0.021)$ & $0.925(0.034)^{*, c}$ & 0.000 & $0.889(0.041)$ & 0.003 & $0.887(0.022)$ & 0.01 \\
\hline $\mathrm{RD}$ & $0.486(0.026)$ & $0.555(0.046)^{*, b}$ & 0.000 & $0.501(0.046)$ & 0.76 & $0.512(0.036)$ & 0.31 \\
\hline \multicolumn{8}{|l|}{ Right } \\
\hline FA & $0.356(0.029)$ & $0.325(0.027)$ & 0.04 & $0.354(0.033)$ & 0.99 & $0.359(0.023)$ & 0.99 \\
\hline MD & $0.591(0.019)$ & $0.647(0.037)^{*}$ & 0.000 & $0.614(0.031)$ & 0.19 & $0.604(0.028)$ & 0.67 \\
\hline$A D$ & $0.836(0.021)$ & $0.889(0.037)^{*}$ & 0.000 & $0.864(0.030)$ & 0.08 & $0.861(0.032)$ & 0.14 \\
\hline RD & $0.464(0.025)$ & $0.524(0.042)^{*}$ & 0.000 & $0.488(0.036)$ & 0.27 & $0.475(0.031)$ & 0.84 \\
\hline \multicolumn{8}{|l|}{ SMAc-Insula } \\
\hline \multicolumn{8}{|l|}{ Left } \\
\hline FA & $0.370(0.030)$ & $0.342(0.038)$ & 0.18 & $0.400(0.039)$ & 0.10 & $0.377(0.032)$ & 0.98 \\
\hline$M D$ & $0.600(0.022)$ & $0.674(0.040)^{*}$ & 0.000 & $0.624(0.039)$ & 0.28 & $0.630(0.026)$ & 0.05 \\
\hline$A D$ & $0.861(0.027)$ & $0.942(0.045)^{*}$ & 0.000 & $0.918(0.050)$ & 0.004 & $0.902(0.023)$ & 0.03 \\
\hline $\mathrm{RD}$ & $0.470(0.028)$ & $0.543(0.042)^{*}$ & 0.000 & $0.479(0.041)$ & 0.98 & $0.491(0.035)$ & 0.36 \\
\hline \multicolumn{8}{|l|}{ Right } \\
\hline FA & $0.367(0.039)$ & $0.340(0.035)$ & 0.14 & $0.381(0.035)$ & 0.62 & $0.375(0.028)$ & 0.90 \\
\hline$M D$ & $0.587(0.019)$ & $0.642(0.044)^{*}$ & 0.000 & $0.614(0.035)$ & 0.11 & $0.600(0.033)$ & 0.42 \\
\hline$A D$ & $0.843(0.030)$ & $0.900(0.047)$ & 0.003 & $0.887(0.031)$ & 0.01 & $0.864(0.041)$ & 0.12 \\
\hline $\mathrm{RD}$ & $0.458(0.023)$ & $0.514(0.045)^{*}$ & 0.000 & $0.476(0.036)$ & 0.61 & $0.464(0.033)$ & 0.90 \\
\hline \multicolumn{8}{|l|}{ BA44-Insula } \\
\hline \multicolumn{8}{|l|}{ Left } \\
\hline FA & $0.297(0.021)$ & $0.261(0.021)$ & 0.003 & $0.280(0.027)$ & 0.35 & $0.292(0.021)$ & 0.94 \\
\hline MD & $0.639(0.020)$ & $0.702(0.033)^{*}$ & 0.000 & $0.675(0.040)$ & 0.05 & $0.660(0.035)$ & 0.25 \\
\hline$A D$ & $0.867(0.024)$ & $0.912(0.032)$ & 0.004 & $0.890(0.034)$ & 0.40 & $0.885(0.039)$ & 0.34 \\
\hline $\mathrm{RD}$ & $0.531(0.020)$ & $0.600(0.034)^{*}$ & 0.000 & $0.569(0.041)$ & 0.03 & $0.551(0.033)$ & 0.25 \\
\hline \multicolumn{8}{|l|}{ Right } \\
\hline FA & $0.295(0.021)$ & $0.275(0.025)$ & 0.21 & $0.284(0.019)$ & 0.40 & $0.301(0.019)$ & 0.99 \\
\hline MD & $0.626(0.020)$ & $0.658(0.035)$ & 0.02 & $0.640(0.019)$ & 0.38 & $0.638(0.022)$ & 0.43 \\
\hline$A D$ & $0.841(0.021)$ & $0.860(0.032)$ & 0.24 & $0.848(0.022)$ & 0.94 & $0.859(0.020)$ & 0.30 \\
\hline $\mathrm{RD}$ & $0.521(0.023)$ & $0.559(0.038)$ & 0.02 & $0.536(0.022)$ & 0.42 & $0.525(0.026)$ & 0.86 \\
\hline \multicolumn{8}{|c|}{ BA6vPMC-Insula } \\
\hline \multicolumn{8}{|c|}{ Left } \\
\hline FA & $0.299(0.023)$ & $0.266(0.029)$ & 0.005 & $0.307(0.015)$ & 0.77 & $0.298(0.016)$ & 0.99 \\
\hline MD & $0.640(0.018)$ & $0.690(0.035)^{*}$ & 0.000 & $0.648(0.031)$ & 0.82 & $0.662(0.022)$ & 0.13 \\
\hline$A D$ & $0.862(0.024)$ & $0.900(0.035)$ & 0.03 & $0.866(0.030)$ & 0.93 & $0.887(0.034)$ & 0.05 \\
\hline $\mathrm{RD}$ & $0.529(0.022)$ & $0.587(0.038)^{*}$ & 0.000 & $0.537(0.027)$ & 0.92 & $0.550(0.022)$ & 0.25 \\
\hline \multicolumn{8}{|l|}{ Right } \\
\hline FA & $0.317(0.018)$ & $0.288(0.025)$ & 0.02 & $0.312(0.024)$ & 0.90 & $0.317(0.029)$ & 0.98 \\
\hline$M D$ & $0.637(0.020)$ & $0.662(0.031)$ & 0.16 & $0.642(0.021)$ & 0.91 & $0.643(0.035)$ & 0.77 \\
\hline$A D$ & $0.863(0.026)$ & $0.873(0.029)$ & 0.95 & $0.865(0.024)$ & 0.51 & $0.874(0.030)$ & 0.97 \\
\hline $\mathrm{RD}$ & $0.522(0.017)$ & $0.556(0.034)$ & 0.03 & $0.529(0.025)$ & 0.88 & $0.527(0.036)$ & 0.83 \\
\hline
\end{tabular}

$M D, A D$, and $R D$ values are reported as mean (SD) in $\mathrm{mm}^{2} / \mathrm{s} \times 10^{-3}$. *Significant differences between PPA variants and healthy controls at $p<0.002$ (Bonferroni adjusted).

${ }^{a}$ Significant differences from nonfluent variants at $p<0.05$.

${ }^{b}$ Significant differences from semantic variants at $p<0.05$.

'Significant differences from logopenic variants at $p<0.05$.

left IFG ( $x=-42, y=17, z=25$; number of voxels $=6)$ survived FWE correction.

\section{Tractography results in healthy controls}

We were able to delineate intrafrontal, frontostriatal, and frontoinsular pathways that connected specific GM regions within the speech production network (Figs. 4, 5, 6). The tracts connecting BA44 and BA6vPMC to SMAc (Fig. 4A) showed the highest consistency among subjects (on average, 100\% for BA44-SMAc and $83 \%$ for BA6vPMC-SMAc). Despite the fact that SMAc was considered as one single ROI, the tracts connecting BA44 and BA6vPMC and SMAc formed two separate pathways with a pref- 
Table 3. Group differences in the left language tracts for all DTI metrics

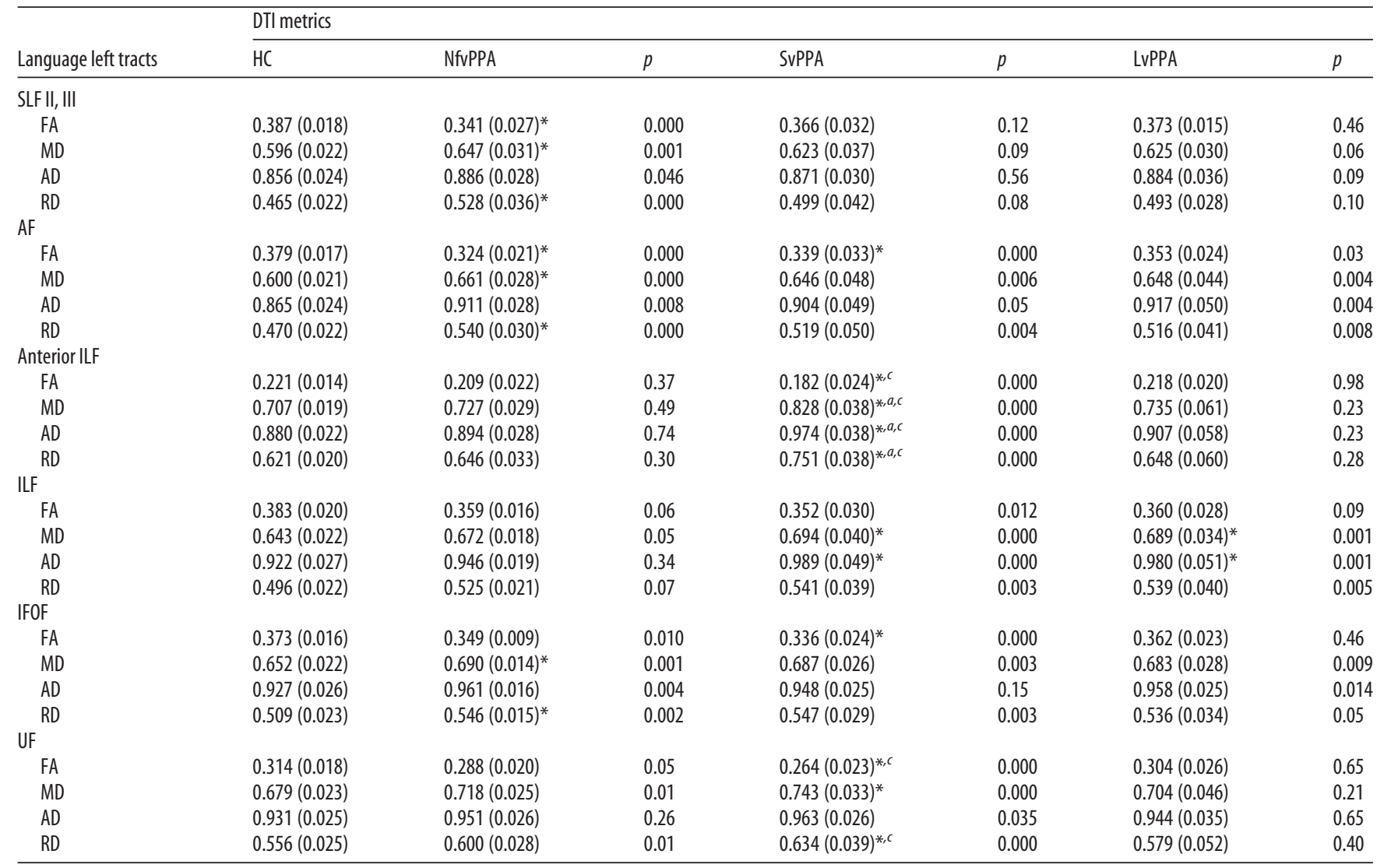

$\mathrm{MD}, \mathrm{AD}$, and $\mathrm{RD}$ values are reported as mean (SD) in $\mathrm{mm}^{2} / \mathrm{s} \times 10^{-3}$. ${ }^{*}$ Significant differences between PPA variants and healthy controls at $p<0.002$ (Bonferroni adjusted).

${ }^{a}$ Significant differences from nonfluent variants at $p<0.05$.

${ }^{b}$ Significant differences from semantic variants at $p<0.05$.

'Significant differences from logopenic variants at $p<0.05$.

erential anterior-posterior distribution: pre-SMA to BA44 (Fig. $4 A$, blue), an area previously described as the aslant tract (Catani et al., 2013), and SMA proper to BA6vPMC (Fig. 4A, green).

We found connections between the insula and BA6vPMC, BA44, and SMAc (Fig. 4B). The most reliable connections were found from the insula to the ventral premotor cortex (vPMC), and SMAc (79\% for BA6vPMC-Insula; 76\% for SMAc-Insula). Even if the whole insula was included as our ROI, we observed that insular projections to BA6vPMC and SMAc originated from a specific portion of the insula's precentral gyrus that, when damaged by stroke, is associated with AOS (Dronkers, 1996). The insular projections to BA44 (52\% for BA44-Insula) appeared instead to terminate more anteriorly in the middle short insular and short insular gyri. We did not find reliable connections between the insular cortex and the striatum, most likely because of anatomical proximity.

We identified projections between the striatum and SMAc (74\% for SMAc-Caudate; $74 \%$ for SMAc-Putamen), and between the striatum and the inferior frontal cortex (71\% for BA44Caudate; $83 \%$ for BA44-Putamen; $40 \%$ for BA6vPMC-Caudate; $64 \%$ for BA6vPMC-Putamen). Tractography to and from the striatum is known to be challenging due to the low FA in this area, and less consistency was expected in particular for the caudate (Lehéricy et al., 2004; Ford et al., 2013). As in the case of the connections between the inferior frontal ROIs and SMAc, the pathway between the striatum and BA44 was positioned more anteriorly than the one originating from the BA6vPMC (Fig. $5 A, B)$. The tracts from BA44, BA6vPMC, and SMAc showed connections to the middle-posterior portion of caudate and putamen (Fig. 5C). This finding is consistent with previous studies showing that the anterior striatum is connected to more prefrontal areas (Lehéricy et al., 2004; Draganski et al., 2008).

In Figure 6, we include all WM tracts connecting the speech production network in healthy subjects, illustrating its highly complex organization.

\section{WM changes in PPA variants}

We compared DTI metrics in 22 bilateral frontal WM pathways (11 for each hemisphere) and five left language tracts (Tables 2, 3).

NfvPPA patients showed significant differences at our threshold in all DTI metrics (decreased FA and increased AD, RD, and $\mathrm{MD})$ relative to controls in the following connections: left BA44SMAc, BA6vPMC-SMAc, BA44-Putamen, and bilateral SMAcCaudate. We consider these tracts as being the most significantly and selectively damaged pathways in nfvPPA, although other tracts also showed changes in two or three DTI metrics. In particular, significant increases in $\mathrm{AD}, \mathrm{RD}$, and MD were observed in tracts connecting left SMAc-Insula, bilateral BA44-Caudate, bilateral BA6vPMC-Caudate, bilateral SMAc-Putamen, and right BA6vPMC-SMAc; significant increases in RD and MD were found in tracts connecting right BA44-SMAc, left BA44-Insula, left BA6vPMC-Insula, right SMAc-Insula, and left BA6vPMCPutamen. Figures 7 and 8 illustrate the distribution of FA and MD in each PPA variant and in controls in those tracts that show changes in FA and all other diffusivity metrics in nfvPPA (BA6SMAc, BA44-SMAc, BA44-Putamen, SMAc-Caudate), tracts 


\section{BA44/BA6-SMAC BA44-Putamen SMAc-Caudate BA44-Caudate ILF}

Healthy controls

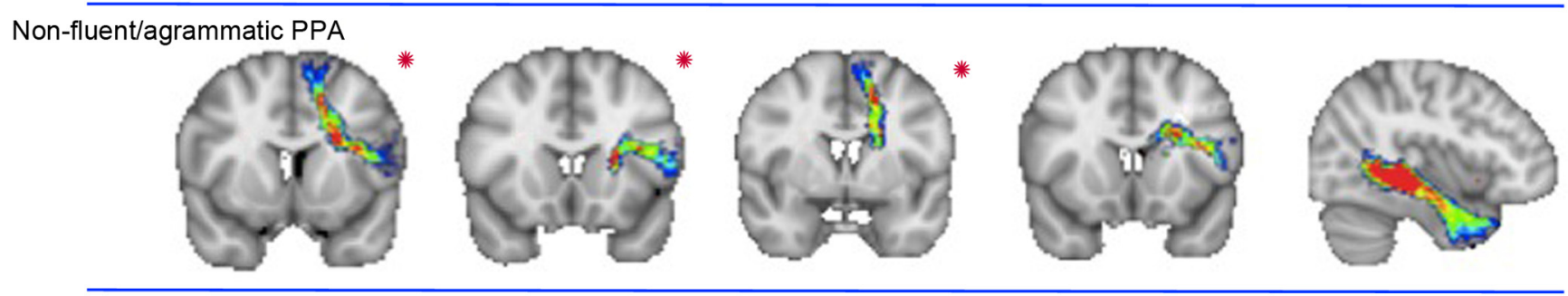

Logopenic PPA
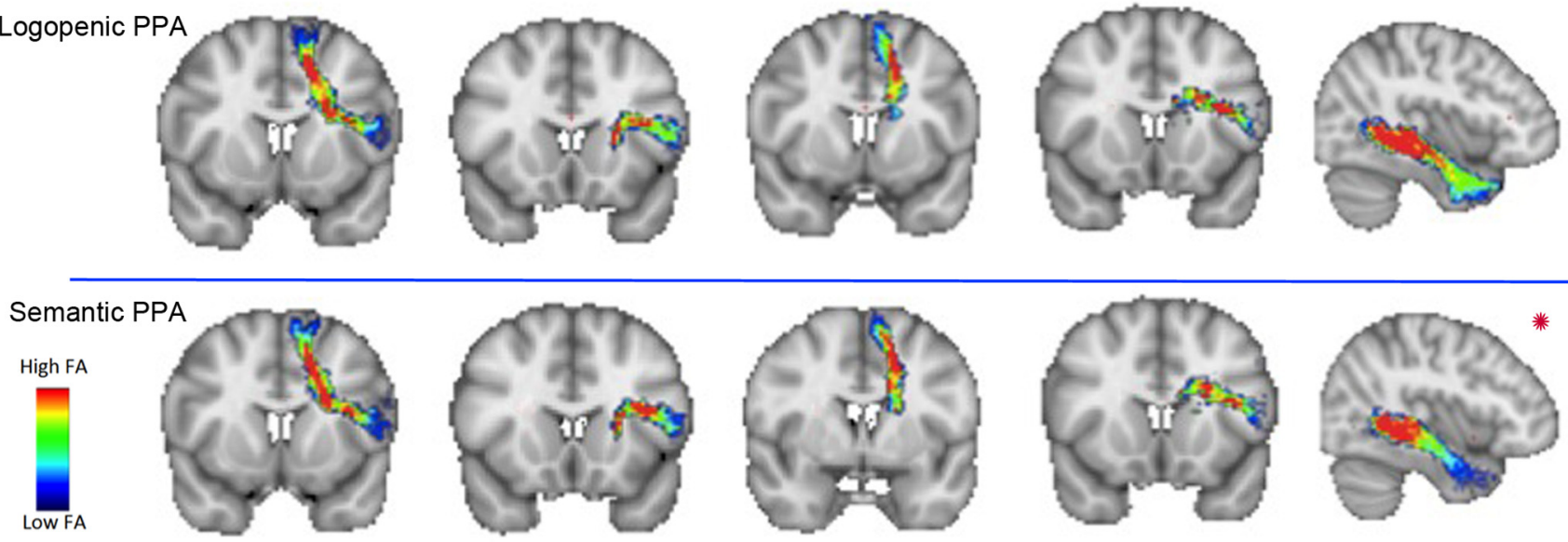

Figure 7. Distribution of fractional anisotropy along the tracts of the speech production network and the ILF. Shown are group averages of the distribution of FA along the speech production tracts statistical significant in the nfvPPA group after multiple comparisons (red asterisk). We also reported a tract of the network (BA44-Caudate) not significant after multiple comparisons for FA but significant in all of the other metrics and the ILF as control tract in nfvPPA. In the scale bar, red indicates regions of the tract with high FA values and blue indicates low FA values. The significance of FA in the ILF was found in SVPPA, mainly in its anterior portion.

where nfvPPA patients exhibited changes in all metrics except FA (BA44-Caudate) and also tracts where no changes were observed (ILF) in nfvPPA.

When considering the standard language tracts, the nfvPPA group showed significant changes in $\mathrm{FA}, \mathrm{MD}$, and $\mathrm{RD}$ in the SLFII/III and AF and in MD and RD in the IFOF. The svPPA and lvPPA groups did not show any significant difference in any of the fronto-insular speech production tracts compared with controls. SvPPA and lvPPA showed changes in the language tracts consistent with other studies (Galantucci et al., 2011; Wilson et al., 2011; Agosta et al., 2012a).

In the comparison among PPA subtypes, we considered only the speech production tracts that were significantly changed in nfvPPA versus controls. All of these tracts showed greater changes in nfvPPA than in svPPA and lvPPA in at least one DTI metric (see Table 2 for details).

\section{Correlation analyses between cognitive scores and tract- specific FA values}

Specificity of the frontal speech versus ventral tracts for speech production and comprehension in PPA

Significant correlations were found between the SP score and FA in left frontal speech tracts that presented the most significant damage in nfvPPA. No correlation was found with the semantic association task or with the word comprehension task. The double dissociation was seen in the anterior ILF that showed significant correlation with the picture and word comprehension tasks but not with the SP score (Fig. 9, Table 4). Although these correlations might in part be driven by group effects in the nfvPPA and svPPA groups, the plots reported in Figure 9 clearly indicated that data from all PPA patients followed the same patterns of distribution. This pattern of correlation indicates specificity of these tracts for speech production versus comprehension mechanisms.

Differential contribution of frontal speech versus language tracts to the cognitive subcomponents of speech production

In this analysis, we investigated further the role of different left frontal speech and standard dorsal language tracts (SLF II/III and $\mathrm{AF}$ ) in speech production mechanisms in the nfvPPA group. For example, only this variant showed variability of performance in the relevant tasks such as number of distortions, rate of speech, and syntax production. Number of distortions (proxy for AOS) correlated with the tracts BA44-SMAc, BA6vPMC-SMAc, and SMAc-Caudate, but not with SLF or AF. Rate of speech correlated with BA44-SMAc, SMAc-Caudate and SLF II/III. Syntax production scores correlated with BA44-SMAc and with both SLF II/III and AF. Sentence comprehension correlated with SLF II/III and $\mathrm{AF}$ and not with the frontal speech tracts (Table 5). These corre- 


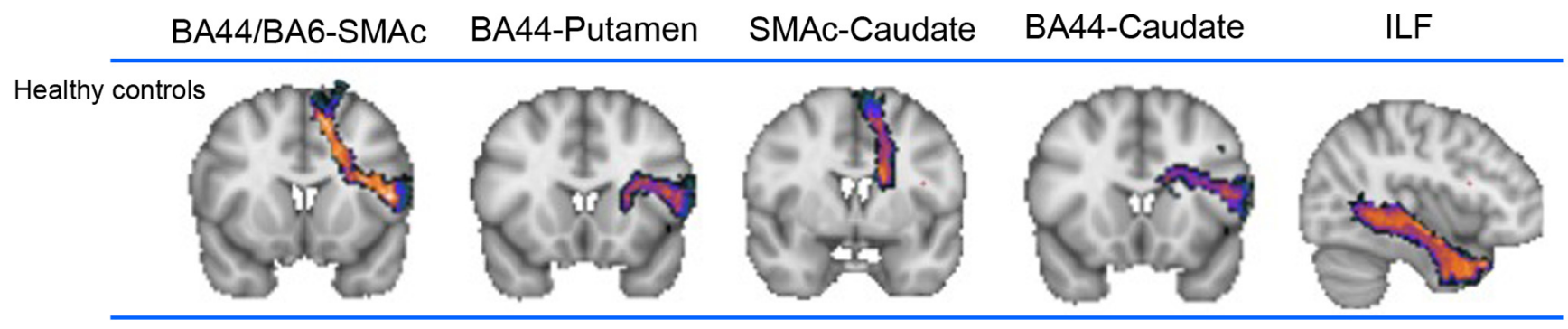

Non-fluent/agrammatic PPA

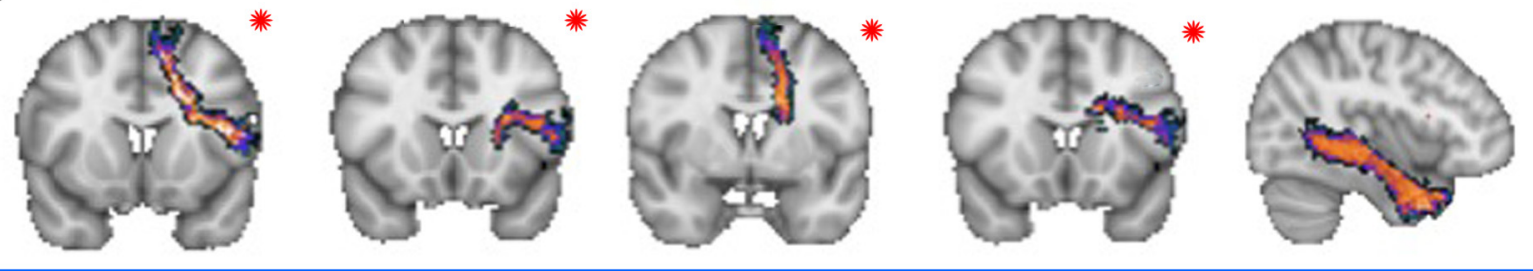

Logopenic PPA
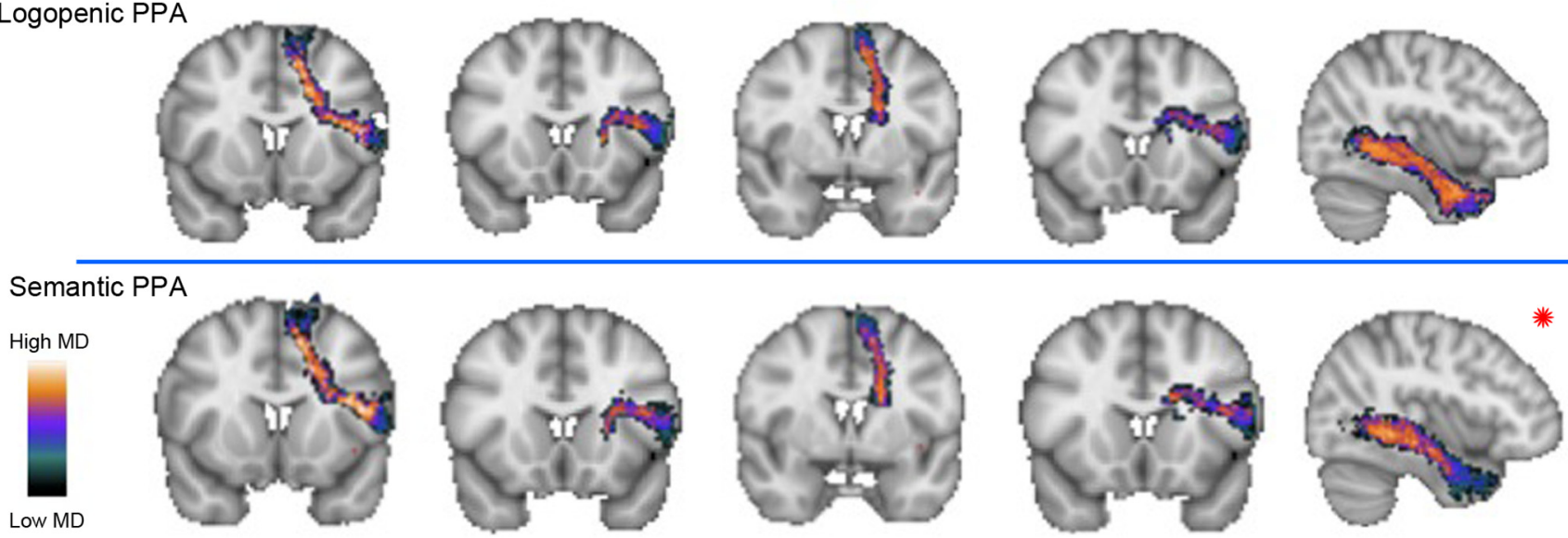

Figure 8. Distribution of mean diffusivity along the tracts of the speech production network and the ILF. Shown are group averages of the distribution of MD along the speech production tracts that were statistically significant in the nfvPPA group after multiple comparisons (red asterisk). We also reported a tract of the network (BA44-Caudate) significant after multiple comparisons for MD, but not in all of the DTI metrics, and the ILF as control tract in nfvPPA. In the scale bar, orange/white indicates high MD and green/black indicates low MD.

lations thus showed a double dissociation between the AOS score (number of distortions), which correlated with frontal speech but not standard dorsal language tracts, and sentence comprehension, which correlated with language but not frontal tracts. Although the number of subjects is small, the presence of a double dissociation, consistency with previous findings, and the coherent distribution demonstrated by the scatterplots in Figure 10 all suggest that these correlations represent a promising finding.

\section{Discussion}

In the present study, we delineated tracts connecting the frontoinsular cortical and subcortical network relevant to speech production and investigated their integrity and functional relevance in the main three PPA variants. We found severe WM damage in tracts connecting left premotor, inferior frontal, SMAc, and the striatum in the nfvPPA variant only. Moreover, correlation analyses confirmed the specific role of these tracts in speech production processes, and provided evidence for their involvement in the motoric aspects of fluency. We discuss these findings in the context of previous MR diffusion studies in PPA, highlighting the anatomical and functional relevance of the frontal tracts for speech production. We argue that converging evidence points to a role for WM imaging in the differential diagnosis of PPA variants and possibly in the in vivo prediction of its pathological underpinnings.
Substantial evidence coming from patients with stroke (Broca, 1865; Dronkers, 1996) and functional neuroimaging studies in healthy controls (Eickhoff et al., 2009) supports the fundamental role of a left fronto-insular cortico-subcortical network of GM regions in speech production. Consistently, neuroimaging studies of GM atrophy in nfvPPA, comprising the VBM results reported here, have shown damage to the same network. Less is known about the WM connections within these regions in healthy controls (Lawes et al., 2008; Oishi et al., 2008; Catani et al., 2012) and even less in PPA (Catani et al., 2013). Here, we provide evidence for widespread cortico-subcortical WM damage in the speech production network in the nfvPPA only, whereas these pathways were relatively spared in the semantic and logopenic variants. Previous DTI studies have highlighted that nfvPPA is associated with a severe degree of WM damage, particularly in the dorsal frontoparietal language pathway (Whitwell et al., 2010; Galantucci et al., 2011; Grossman, 2012; Grossman et al., 2013; Mahoney et al., 2013; Sajjadi et al., 2013; Schwindt et al., 2013). However, these studies agree that DTI changes in dorsal language tracts are not specific to nfvPPA, but are also present in lvPPA (Galantucci et al., 2011; Agosta et al., 2013). Our new results show that the WM pathways within the frontal speech production network might be a better anatomical 
BA44/BA6-SMAc L
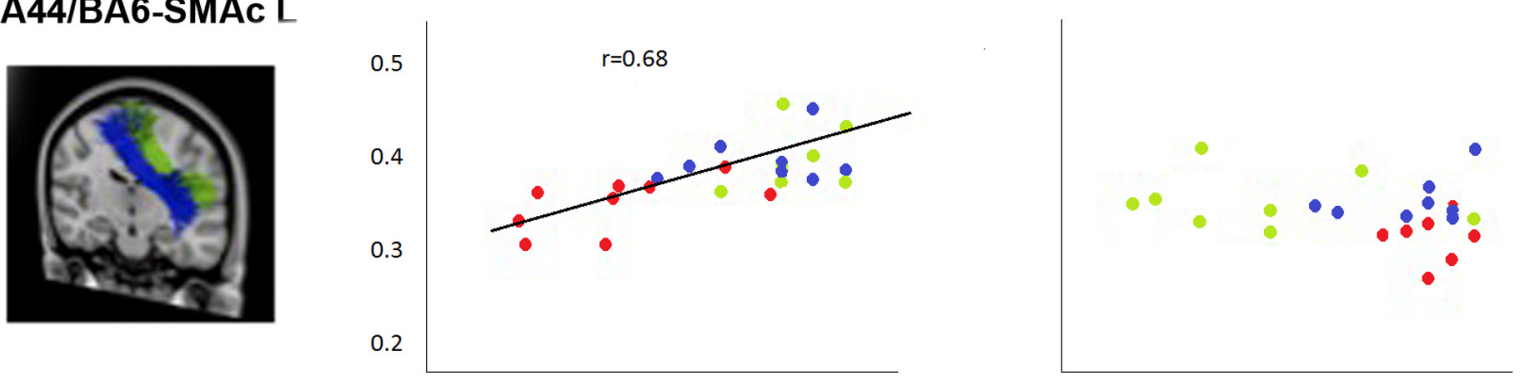

\section{SMAc-Caudate L}
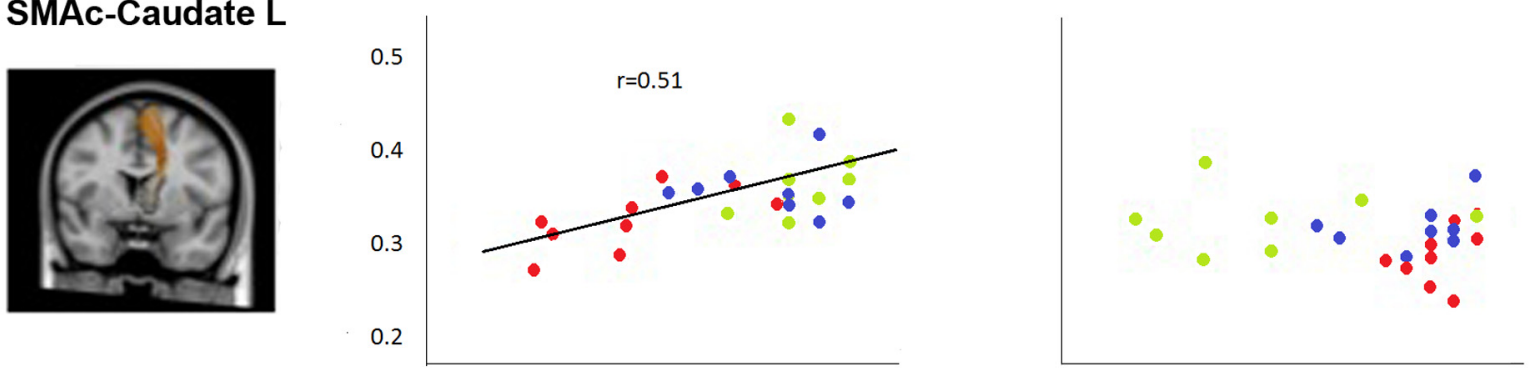

\section{Anterior ILF L}
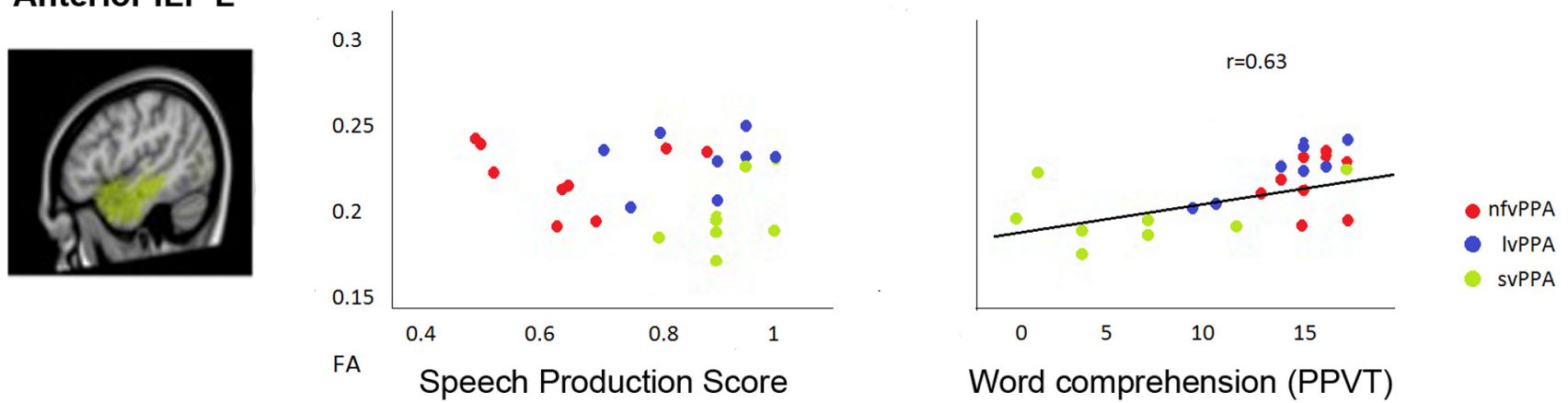

Figure 9. Correlations in PPA between the speech production score and the picture vocabulary test with FA in frontal speech tracts and the anterior ILF (alLF). FA correlates with the speech production score in the frontal speech tracts (left BA44/BA6vPMC-SMAc and SMAC-Caudate) and not with the picture vocabulary test. FA correlates with the picture vocabulary test in the alLF and not with the speech production score. The different colors in the scatterplots represent the three variants: red for nfvPPA, blue for IvPPA, and green for svPPA.

Table 4. Correlations between speech production and semantic/comprehension tests and FA in the frontal speech and ventral language tracts in PPA

\begin{tabular}{|c|c|c|c|c|c|c|}
\hline \multirow[b]{2}{*}{ FA } & \multicolumn{2}{|c|}{$\begin{array}{l}\text { Speech } \\
\text { production } \\
\text { score }\end{array}$} & \multicolumn{2}{|c|}{$\begin{array}{l}\text { Semantic } \\
\text { association } \\
\text { (PPTP) }\end{array}$} & \multicolumn{2}{|c|}{$\begin{array}{l}\text { Word } \\
\text { comprehension } \\
\text { (PPVT) }\end{array}$} \\
\hline & $\rho$ & $p$ & $\rho$ & $p$ & $\rho$ & $p$ \\
\hline \multicolumn{7}{|c|}{ Left frontal speech tracts } \\
\hline $\begin{array}{c}\text { BA44-SMAc } \\
\text { (pre-SMA) }\end{array}$ & 0.72 & $0.000^{*}$ & -0.03 & 0.87 & -0.15 & 0.46 \\
\hline $\begin{array}{r}\text { BA6vPMC-SMAC } \\
\text { (SMA proper) }\end{array}$ & 0.56 & $0.004^{*}$ & -0.11 & 0.58 & -0.38 & 0.06 \\
\hline BA44-Putamen & 0.57 & $0.003^{*}$ & 0.22 & 0.28 & 0.07 & 0.74 \\
\hline SMAc-Caudate & 0.51 & $0.008^{*}$ & -0.06 & 0.78 & 0.04 & 0.85 \\
\hline \multicolumn{7}{|c|}{ Left ventral language tracts } \\
\hline Anterior ILF & -0.08 & 0.67 & 0.72 & $0.000^{*}$ & 0.63 & $0.000^{*}$ \\
\hline ILF & 0.08 & 0.68 & 0.35 & 0.08 & 0.40 & $0.045^{*}$ \\
\hline
\end{tabular}

*Significant correlations in PPA variants at $p<0.05$.

PPTP, Pyramids and Palm Trees Test-Picture Version; PPVT, Peabody Picture Vocabulary Test-Revised.

location for futures studies investigating DTI as a specific nfvPPA biomarker. One previous study found changes in what was called the frontal aslant tract (Catani et al., 2013), which connects the opercular portion of the left inferior frontal gyrus and a medial frontal area including preSMA, anterior SMA, and anterior cin- gulate cortices. Although direct anatomical comparison is difficult, the aslant tract likely includes both our BA44 and ventral BA6 to SMAc tracts, showing consistency between the two studies. Here, we found that, in nfvPPA, WM damage was not limited to this intrafrontal pathway, but was more widespread and included the left frontostriatal tracts originating from both inferior frontal (BA6 and BA44) and SMAc cortical regions. Furthermore, although these fiber bundles were the most significantly damaged (corrected for multiple comparisons in all DTI metrics), all left hemisphere pathways connecting our ROIs (Figs. 1, 3 ) showed at least a trend for damage in the nfvPPA group only. In addition, although the specific connections between the left precentral insula and inferior and medial frontal regions did not reach a corrected level of significance in all DTI metrics, they were nevertheless widely damaged in our nfvPPA patients with AOS (Dronkers, 1996). These results, showing widespread speech production system damage in nfvPPA, are consistent with recent pathophysiological models of network-specific susceptibility to neurodegeneration (Zhou et al., 2010). Recent neuroimaging results and neurobiological evidence indicate that neurodegeneration occurring in specific cortical hubs then spreads to structurally and functionally connected regions (Raj et al., 2012) through prion-like transsynaptic transmission (Frost et al., 
Table 5. Correlation between scores in subcomponents of fluency and FA in the frontal speech and dorsal language tracts in nfvPPA

\begin{tabular}{|c|c|c|c|c|c|c|c|c|}
\hline \multirow[b]{2}{*}{ FA } & \multicolumn{2}{|c|}{ No. of distortions } & \multicolumn{2}{|c|}{ Speech rate (WPM) } & \multicolumn{2}{|c|}{ Syntax production score } & \multicolumn{2}{|c|}{ Sentence comprehension } \\
\hline & $\rho$ & $p$ & $\rho$ & $p$ & $\rho$ & $p$ & $\rho$ & $p$ \\
\hline \multicolumn{9}{|l|}{ Left frontal speech tracts } \\
\hline BA44-SMAC (pre-SMA) & -0.78 & $0.01^{*}$ & 0.83 & $0.005^{*}$ & 0.76 & $0.02^{*}$ & 0.46 & 0.21 \\
\hline BA6VPMC-SMAC (SMA proper) & -0.70 & $0.03^{*}$ & 0.50 & 0.17 & 0.27 & 0.48 & 0.10 & 0.79 \\
\hline BA44-Putamen & -0.27 & 0.49 & 0.45 & 0.22 & 0.45 & 0.22 & 0.47 & 0.20 \\
\hline SMAc-Caudate & -0.72 & $0.03^{*}$ & 0.75 & $0.02^{*}$ & 0.65 & 0.06 & 0.28 & 0.45 \\
\hline \multicolumn{9}{|l|}{ Left dorsal language tracts } \\
\hline SLF II/III & -0.65 & 0.06 & 0.78 & $0.01^{*}$ & 0.76 & $0.02^{*}$ & 0.78 & $0.01^{*}$ \\
\hline $\mathrm{AF}$ & -0.37 & 0.33 & 0.53 & 0.13 & 0.76 & $0.02^{*}$ & 0.84 & $0.004^{*}$ \\
\hline
\end{tabular}

*Significant correlations in PPA variants at $p<0.05$.

\section{BA44/BA6-SMAc L}
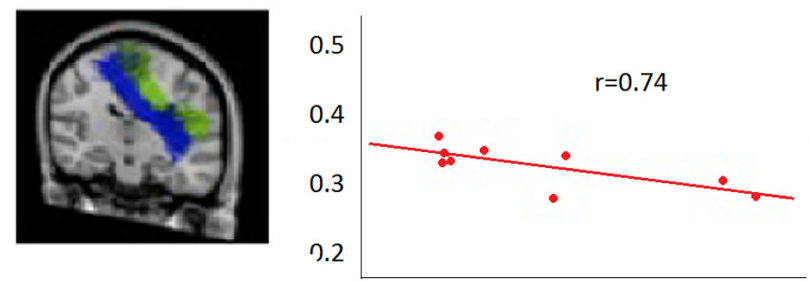
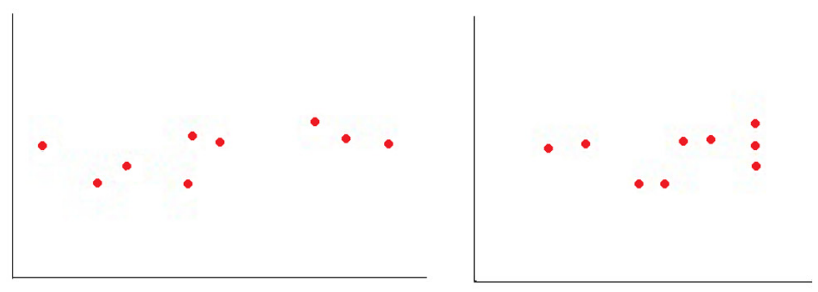

\section{Arcuate Fasciculus L}

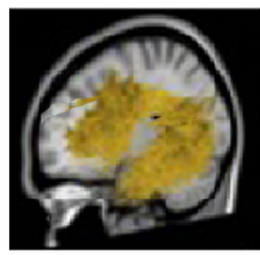

nfvPPA

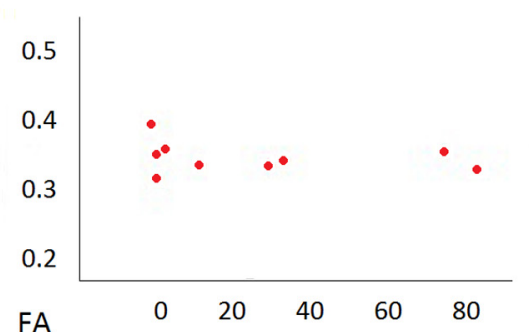

Number of Distortions

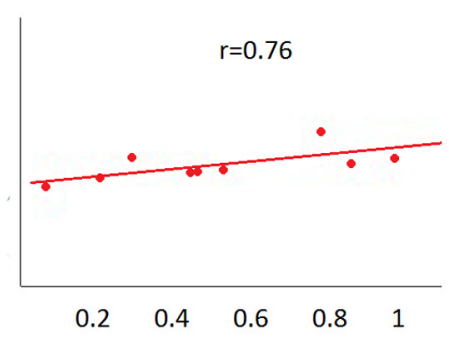

Syntax Production Score

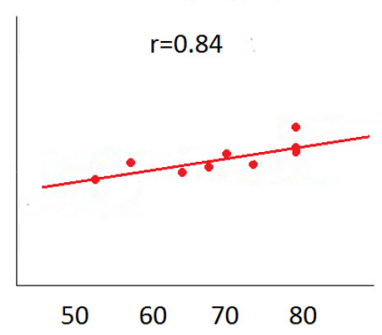

Sentence Comprehension

Figure 10. Correlations in nfvPPA between number of distortions, syntax production score, and sentence comprehension with FA in frontal speech tracts and the AF. FA correlates with number of distortions in the left BA44/BA6vPMC-SMAc, but not with syntax production score and sentence comprehension. FA correlates with syntax production score and sentence comprehension in the left arcuate fasciculus, but not with the number of distortions.

2009). Pathological changes underlying the sporadic form of nfvPPA are most often FTLD-4Rtau or, less commonly, FTLDTDP-A (Josephs et al., 2006; Mesulam et al., 2008; Yokota et al., 2009; Grossman, 2010). With nfvPPA, one could speculate that the tau or TDP protein changes first occur in the ventral premotor and posterior Broca's area and then spread to connected SMA, insula, and striatal circuits and, as disease progresses, to posterior dorsal language areas. In a small clinicopathological correlation study on a homogeneous UCSF cohort of sporadic nfvPPA, we observed that WM atrophy was most prominent in tau compared with TDP-A (Caso et al., 2014). This was also found in studies comparing heterogeneous patient populations with tau versus other types of pathology (McMillan et al., 2013). Considering that tau-related pathological changes are severe in glial WM (Dickson et al., 2002; Zhukareva et al., 2006), diffusion imaging is emerging as a potentially useful biomarker in the complex task of predicting the molecules underlying frontotemporal dementia-spectrum clinical syndromes comprising nfvPPA.

Our results provide evidence regarding the anatomical distribution and functional relevance of the speech production network WM tracts. We found that the so-called "aslant" pathway contains two components: a more anterior projection connecting BA44 with pre-SMA (Fig. 4A; Lawes et al., 2008; Oishi et al., 2008;
Ford et al., 2010; Thiebaut de Schotten et al., 2012) and a more posterior component connecting the ventral premotor area associated with the face/mouth (BA6vPMC), mostly with SMA proper (Fig. 4B). Connections between the inferior frontal region and striatum also followed a rostrocaudal distribution, with fibers connecting BA44 being positioned more anteriorly that those originating from premotor cortex (Fig. 5C; Johansen-Berg et al., 2004; Lehéricy et al., 2004; Leh et al., 2007; Zhang et al., 2012). Previous monkey and human neurophysiological and neuroimaging studies support a role of this frontostriatal network in motor behavior, with more posterior regions (SMApremotor-anterior striatum) involved in the initiation and execution of simple movements and a more anterior system (preSMA-BA44) responsible for more complex tasks such as learning and retrieval of motor sequences (Chung et al., 2005; Alario et al., 2006; Nachev et al., 2008; Kim et al., 2010). A similar anatomo-functional division of the frontostriatal regions has been postulated for language-related tasks, with SMA proper being responsible for execution of speech movements and preSMA in higher-order aspects of speech such as word selection and sequencing (Chung et al., 2005; Alario et al., 2006; Nachev et al., 2008; Kim et al., 2010). Information regarding word and sentence meaning is instead thought to be computed in ventral language 
areas and likely conveyed to frontal regions through the dorsal WM pathways, including the superior longitudinal and arcuate fasciculi (Hickok and Poeppel, 2007; Patterson et al., 2007; Wilson et al., 2011). Within this framework (see also Fig. 2), our brain-behavioral correlations provided evidence regarding the specific functional role of left hemisphere speech and language pathways. First, we showed a double dissociation between left frontal speech tracts and ventral pathways by demonstrating that a general measure of speech production abilities (or fluency) and semantic tasks correlated with left frontal speech tracts and left inferior longitudinal fasciculus respectively. Catani et al. (2013) showed a similar double dissociation between verbal fluency and semantic processing in the aslant and uncinate fasciculus. Taking advantage of the extensive cognitive and DTI data that were collected and processed, we were also able to investigate the differential role of WM pathways in sustaining specific aspects of "fluency" in nfvPPA, such as motor speech, grammar production, and comprehension. Here, we were able to show that the left posterior connections between SMA and ventral premotor correlated only with the number of distortions, a proxy for AOS errors (see Materials and Methods for details). Conversely, the more anterior component of the frontal aslant, connecting posterior Broca's (BA44) with anterior SMA (preSMA), correlated with higher level tasks such as syntax production, consistent with the posited role of preSMA in word sequencing (Chung et al., 2005; Alario et al., 2006; Nachev et al., 2008; Kim et al., 2010). Finally, sentence comprehension abilities in nfvPPA correlated with left frontoparietal (SLF) and frontotemporal $(\mathrm{AF})$, but not intrafrontal tract integrity, likely due to the linguistic complexity of this task (Caplan, 1998; Wilson et al., 2010). These results contribute to the understanding of the anatomical basis of speech and language functions within a large, distributed left-hemisphere network.

The main limitations of our study are the relatively small number of cases included and the lack of pathological confirmation. Interpretation of WM damage as an indication of pathological subtype therefore remains speculative; however, congruence with a priori hypotheses and consistency with previous findings mitigate these concerns.

In conclusion, we demonstrated that damage to WM tracts within the left frontostriatal speech production network is specific to nfvPPA only and that WM damage contributes to the patients' motor speech and grammar production deficits. These findings provide novel evidence for the differential diagnosis of the PPA variants and increase our knowledge of the specific role of WM tracts in speech and language functions.

\section{References}

Agosta F, Canu E, Sarro L, Comi G, Filippi M (2012a) Neuroimaging findings in frontotemporal lobar degeneration spectrum of disorders. Cortex 48:389-413. CrossRef Medline

Agosta F, Scola E, Canu E, Marcone A, Magnani G, Sarro L, Copetti M, Caso F, Cerami C, Comi G, Cappa SF, Falini A, Filippi M (2012b) White matter damage in frontotemporal lobar degeneration spectrum. Cereb Cortex 22:2705-2714. CrossRef Medline

Agosta F, Galantucci S, Canu E, Cappa SF, Magnani G, Franceschi M, Falini A, Comi G, Filippi M (2013) Disruption of structural connectivity along the dorsal and ventral language pathways in patients with nonfluent and semantic variant primary progressive aphasia: a DT MRI study and a literature review. Brain Lang 127:157-166. CrossRef Medline

Alario FX, Chainay H, Lehéricy S, Cohen L (2006) The role of the supplementary motor area (SMA) in word production. Brain Res 1076:129-143. CrossRef Medline

Ashburner J (2007) A fast diffeomorphic image registration algorithm. Neuroimage 38:95-113. CrossRef Medline

Berman JI, Chung S, Mukherjee P, Hess CP, Han ET, Henry RG (2008)
Probabilistic streamline q-ball tractography using the residual bootstrap. Neuroimage 39:215-222. CrossRef Medline

Borroni B, Brambati SM, Agosti C, Gipponi S, Bellelli G, Gasparotti R, Garibotto V, Di Luca M, Scifo P, Perani D, Padovani A (2007) Evidence of white matter changes on diffusion tensor imaging in frontotemporal dementia. Arch Neurol 64:246-251. CrossRef Medline

Broca P (1865) Sur la faculte du langage articule. Bull Soc Anthrop Paris 6:337-393.

Caplan DH, H (1998) Disorders of syntactic comprehension. Cambridge: MIT.

Caso F, Mandelli ML, Henry M, Gesierich B, Bettcher BM, Ogar J, Filippi M, Comi G, Magnani G, Sidhu M, Trojanowski JQ, Huang EJ, Grinberg LT, Miller BL, Dronkers N, Seeley WW, Gorno-Tempini ML (2014) In vivo signatures of nonfluent/agrammatic primary progressive aphasia caused by FTLD pathology. Neurology 82:239-247. CrossRef Medline

Catani M, Dell'acqua F, Vergani F, Malik F, Hodge H, Roy P, Valabregue R, Thiebaut de Schotten M (2012) Short frontal lobe connections of the human brain. Cortex 48:273-291. CrossRef Medline

Catani M, Mesulam MM, Jakobsen E, Malik F, Martersteck A, Wieneke C, Thompson CK, Thiebaut de Schotten M, Dell'Acqua F, Weintraub S, Rogalski E (2013) A novel frontal pathway underlies verbal fluency in primary progressive aphasia. Brain 136:2619-2628. CrossRef Medline

Chung GH, Han YM, Jeong SH, Jack CR Jr (2005) Functional heterogeneity of the supplementary motor area. AJNR Am J Neuroradiol 26:1819-1823. Medline

Dickson DW1, Bergeron C, Chin SS, Duyckaerts C, Horoupian D, Ikeda K, Jellinger K, Lantos PL, Lippa CF, Mirra SS, Tabaton M, Vonsattel JP, Wakabayashi K, Litvan I; Office of Rare Diseases of the National Institutes of Health (2002) Office of Rare Diseases neuropathologic criteria for corticobasal degeneration. J Neuropathol Exp Neurol 61:935-946. Medline

Draganski B, Kherif F, Klöppel S, Cook PA, Alexander DC, Parker GJ, Deichmann R, Ashburner J, Frackowiak RS (2008) Evidence for segregated and integrative connectivity patterns in the human basal ganglia. J Neurosci 28:7143-7152. CrossRef Medline

Dronkers NF (1996) A new brain region for coordinating speech articulation. Nature 384:159-161. CrossRef Medline

Eickhoff SB, Heim S, Zilles K, Amunts K (2009) A systems perspective on the effective connectivity of overt speech production. Philos Trans A Math Phys Eng Sci 367:2399-2421. CrossRef Medline

Ford A, McGregor KM, Case K, Crosson B, White KD (2010) Structural connectivity of Broca's area and medial frontal cortex. Neuroimage 52: 1230-1237. CrossRef Medline

Ford AA, Triplett W, Sudhyadhom A, Gullett J, McGregor K, Fitzgerald DB, Mareci T, White K, Crosson B (2013) Broca's area and its striatal and thalamic connections: a diffusion-MRI tractography study. Front Neuroanat 7:8. CrossRef Medline

Frost B, Jacks RL, Diamond MI (2009) Propagation of tau misfolding from the outside to the inside of a cell. J Biol Chem 284:12845-12852. CrossRef Medline

Galantucci S, Tartaglia MC, Wilson SM, Henry ML, Filippi M, Agosta F, Dronkers NF, Henry RG, Ogar JM, Miller BL, Gorno-Tempini ML (2011) White matter damage in primary progressive aphasias: a diffusion tensor tractography study. Brain 134:3011-3029. CrossRef Medline

Gorno-Tempini ML, Murray RC, Rankin KP, Weiner MW, Miller BL (2004a) Clinical, cognitive and anatomical evolution from nonfluent progressive aphasia to corticobasal syndrome: a case report. Neurocase 10:426-436. CrossRef Medline

Gorno-Tempini ML, Dronkers NF, Rankin KP, Ogar JM, Phengrasamy L, Rosen HJ, Johnson JK, Weiner MW, Miller BL (2004b) Cognition and anatomy in three variants of primary progressive aphasia. Ann Neurol 55:335-346. CrossRef Medline

Gorno-Tempini ML, Ogar JM, Brambati SM, Wang P, Jeong JH, Rankin KP, Dronkers NF, Miller BL (2006) Anatomical correlates of early mutism in progressive nonfluent aphasia. Neurology 67:1849-1851. CrossRef Medline

Gorno-Tempini ML, Brambati SM, Ginex V, Ogar J, Dronkers NF, Marcone A, Perani D, Garibotto V, Cappa SF, Miller BL (2008) The logopenic/ phonological variant of primary progressive aphasia. Neurology 71:12271234. CrossRef Medline

Gorno-Tempini ML, Hillis AE, Weintraub S, Kertesz A, Mendez M, Cappa SF, Ogar JM, Rohrer JD, Black S, Boeve BF, Manes F, Dronkers NF, Vandenberghe R, Rascovsky K, Patterson K, Miller BL, Knopman DS, Hodges JR, Mesulam MM, Grossman M (2011) Classification of pri- 
mary progressive aphasia and its variants. Neurology 76:1006-1014. CrossRef Medline

Grossman M (2010) Primary progressive aphasia: clinicopathological correlations. Nat Rev Neurol 6:88-97. CrossRef Medline

Grossman M (2011) Biomarkers to identify the pathological basis for frontotemporal lobar degeneration. J Mol Neurosci 45:366-371. CrossRef Medline

Grossman M (2012) The non-fluent/agrammatic variant of primary progressive aphasia. Lancet Neurol 11:545-555. CrossRef Medline

Grossman M, Mickanin J, Onishi K, Hughes E, D'Esposito M, Ding XS, Alavi A, Reivich M (1996) Progressive nonfluent aphasia: language, cognitive, and PET measures contrasted with probable Alzheimer's disease. J Cogn Neurosci 8:135-154. CrossRef Medline

Grossman M, Powers J, Ash S, McMillan C, Burkholder L, Irwin D, Trojanowski JQ (2013) Disruption of large-scale neural networks in nonfluent/agrammatic variant primary progressive aphasia associated with frontotemporal degeneration pathology. Brain Lang 127:106-120. CrossRef Medline

Henry RG, Berman JI, Nagarajan SS, Mukherjee P, Berger MS (2004) Subcortical pathways serving cortical language sites: initial experience with diffusion tensor imaging fiber tracking combined with intraoperative language mapping. Neuroimage 21:616-622. CrossRef Medline

Hickok G, Poeppel D (2007) The cortical organization of speech processing. Nat Rev Neurosci 8:393-402. CrossRef Medline

Hillis AE, Work M, Barker PB, Jacobs MA, Breese EL, Maurer K (2004) Re-examining the brain regions crucial for orchestrating speech articulation. Brain 127:1479-1487. CrossRef Medline

Johansen-Berg H, Behrens TE, Robson MD, Drobnjak I, Rushworth MF, Brady JM, Smith SM, Higham DJ, Matthews PM (2004) Changes in connectivity profiles define functionally distinct regions in human medial frontal cortex. Proc Natl Acad Sci U S A 101:13335-13340. CrossRef Medline

Josephs KA, Petersen RC, Knopman DS, Boeve BF, Whitwell JL, Duffy JR, Parisi JE, Dickson DW (2006) Clinicopathologic analysis of frontotemporal and corticobasal degenerations and PSP. Neurology 66:41-48. CrossRef Medline

Josephs KA, Duffy JR, Strand EA, Machulda MM, Senjem ML, Master AV, Lowe VJ, Jack CR Jr, Whitwell JL (2012) Characterizing a neurodegenerative syndrome: primary progressive apraxia of speech. Brain 135:15221536. CrossRef Medline

Kertesz A (1980) Western aphasia battery. London, Ontario: University of Western Ontario.

Kim JH, Lee JM, Jo HJ, Kim SH, Lee JH, Kim ST, Seo SW, Cox RW, Na DL, Kim SI, Saad ZS (2010) Defining functional SMA and pre-SMA subregions in human MFC using resting state fMRI: functional connectivity-based parcellation method. Neuroimage 49:2375-2386. CrossRef Medline

Lawes IN, Barrick TR, Murugam V, Spierings N, Evans DR, Song M, Clark CA (2008) Atlas-based segmentation of white matter tracts of the human brain using diffusion tensor tractography and comparison with classical dissection. Neuroimage 39:62-79. CrossRef Medline

Leh SE, Ptito A, Chakravarty MM, Strafella AP (2007) Fronto-striatal connections in the human brain: a probabilistic diffusion tractography study. Neurosci Lett 419:113-118. CrossRef Medline

Lehéricy S, Ducros M, Van de Moortele PF, Francois C, Thivard L, Poupon C, Swindale N, Ugurbil K, Kim DS (2004) Diffusion tensor fiber tracking shows distinct corticostriatal circuits in humans. Ann Neurol 55:522-529. CrossRef Medline

Mahoney CJ, Malone IB, Ridgway GR, Buckley AH, Downey LE, Golden HL, Ryan NS, Ourselin S, Schott JM, Rossor MN, Fox NC, Warren JD (2013) White matter tract signatures of the progressive aphasias. Neurobiol Aging 34:1687-1699. CrossRef Medline

McMillan CT, Irwin DJ, Avants BB, Powers J, Cook PA, Toledo JB, McCarty Wood E, Van Deerlin VM, Lee VM, Trojanowski JQ, Grossman M (2013) White matter imaging helps dissociate tau from TDP-43 in frontotemporal lobar degeneration. J Neurol Neurosurg Psychiatry 84:949955. CrossRef Medline

Mesulam MM (1982) Slowly progressive aphasia without generalized dementia. Ann Neurol 11:592-598. CrossRef Medline

Mesulam M, Wicklund A, Johnson N, Rogalski E, Léger GC, Rademaker A, Weintraub S, Bigio EH (2008) Alzheimer and frontotemporal pathology in subsets of primary progressive aphasia. Ann Neurol 63:709-719. CrossRef Medline

Nachev P, Kennard C, Husain M (2008) Functional role of the supplemen- tary and pre-supplementary motor areas. Nat Rev Neurosci 9:856-869. CrossRef Medline

Nestor PJ, Graham NL, Fryer TD, Williams GB, Patterson K, Hodges JR (2003) Progressive non-fluent aphasia is associated with hypometabolism centred on the left anterior insula. Brain 126:2406-2418. CrossRef Medline

Ogar JM, Dronkers NF, Brambati SM, Miller BL, Gorno-Tempini ML (2007) Progressive nonfluent aphasia and its characteristic motor speech deficits. Alzheimer Dis Assoc Disord 21:S23-30. CrossRef Medline

Oishi K, Zilles K, Amunts K, Faria A, Jiang H, Li X, Akhter K, Hua K, Woods R, Toga AW, Pike GB, Rosa-Neto P, Evans A, Zhang J, Huang H, Miller MI, van Zijl PC, Mazziotta J, Mori S (2008) Human brain white matter atlas: identification and assignment of common anatomical structures in superficial white matter. Neuroimage 43:447-457. CrossRef Medline

Patterson K, Nestor PJ, Rogers TT (2007) Where do you know what you know? The representation of semantic knowledge in the human brain. Nat Rev Neurosci 8:976-987. CrossRef Medline

Raj A, Kuceyeski A, Weiner M (2012) A network diffusion model of disease progression in dementia. Neuron 73:1204-1215. CrossRef Medline

Rogalski E, Cobia D, Harrison TM, Wieneke C, Weintraub S, Mesulam MM (2011a) Progression of language decline and cortical atrophy in subtypes of primary progressive aphasia. Neurology 76:1804-1810. CrossRef Medline

Rogalski E, Cobia D, Harrison TM, Wieneke C, Thompson CK, Weintraub S, Mesulam MM (2011b) Anatomy of language impairments in primary progressive aphasia. J Neurosci 31:3344-3350. CrossRef Medline

Sajjadi SA, Acosta-Cabronero J, Patterson K, Diaz-de-Grenu LZ, Williams GB, Nestor PJ (2013) Diffusion tensor magnetic resonance imaging for single subject diagnosis in neurodegenerative diseases. Brain 136:22532261. CrossRef Medline

Schwindt GC, Graham NL, Rochon E, Tang-Wai DF, Lobaugh NJ, Chow TW, Black SE (2013) Whole-brain white matter disruption in semantic and nonfluent variants of primary progressive aphasia. Hum Brain Mapp 34: 973-984. CrossRef Medline

Thiebaut de Schotten M, Dell'Acqua F, Valabregue R, Catani M (2012) Monkey to human comparative anatomy of the frontal lobe association tracts. Cortex 48:82-96. CrossRef Medline

Wertz RT, LaPointe LL, Rosenbek JC (1984) Apraxia of speech: the disorders and its management. New York: Grune and Stratton.

Whitwell JL, Avula R, Senjem ML, Kantarci K, Weigand SD, Samikoglu A, Edmonson HA, Vemuri P, Knopman DS, Boeve BF, Petersen RC, Josephs KA, Jack CR Jr (2010) Gray and white matter water diffusion in the syndromic variants of frontotemporal dementia. Neurology 74:12791287. CrossRef Medline

Wilson SM, Isenberg AL, Hickok G (2009) Neural correlates of word production stages delineated by parametric modulation of psycholinguistic variables. Hum Brain Mapp 30:3596-3608. CrossRef Medline

Wilson SM, Henry ML, Besbris M, Ogar JM, Dronkers NF, Jarrold W, Miller BL, Gorno-Tempini ML (2010) Connected speech production in three variants of primary progressive aphasia. Brain 133:2069-2088. CrossRef Medline

Wilson SM, Galantucci S, Tartaglia MC, Rising K, Patterson DK, Henry ML, Ogar JM, DeLeon J, Miller BL, Gorno-Tempini ML (2011) Syntactic processing depends on dorsal language tracts. Neuron 72:397-403. CrossRef Medline

Yokota O, Tsuchiya K, Arai T, Yagishita S, Matsubara O, Mochizuki A, Tamaoka A, Kawamura M, Yoshida H, Terada S, Ishizu H, Kuroda S, Akiyama H (2009) Clinicopathological characterization of Pick's disease versus frontotemporal lobar degeneration with ubiquitin/ TDP-43-positive inclusions. Acta Neuropathol 117:429-444. CrossRef Medline

Zhang S, Ide JS, Li CS (2012) Resting-state functional connectivity of the medial superior frontal cortex. Cereb Cortex 22:99-111. CrossRef Medline

Zhou J, Greicius MD, Gennatas ED, Growdon ME, Jang JY, Rabinovici GD, Kramer JH, Weiner M, Miller BL, Seeley WW (2010) Divergent network connectivity changes in behavioural variant frontotemporal dementia and Alzheimer's disease. Brain 133:1352-1367. CrossRef Medline

Zhukareva V, Joyce S, Schuck T, Van Deerlin V, Hurtig H, Albin R, Gilman S, Chin S, Miller B, Trojanowski JQ, Lee VM (2006) Unexpected abundance of pathological tau in progressive supranuclear palsy white matter. Ann Neurol 60:335-345. CrossRef Medline 